\title{
MODULI OF GENERALIZED LINE BUNDLES ON A RIBBON
}

\author{
DAWEI CHEN AND JESSE LEO KASS
}

\begin{abstract}
A ribbon is a first-order thickening of a non-singular curve. We show that a compactification of the moduli space of generalized line bundles on a ribbon is given by the Simpson moduli space of semi-stable sheaves. We then describe the geometry of this space, determining the irreducible components, connectedness, and the smooth locus.
\end{abstract}

\section{Contents}

1. Introduction 1

2. Coherent Sheaves on a Ribbon 5

3. Stable Sheaves on a Ribbon 12

4. Moduli of Sheaves on a Ribbon 16

4.1. Global Structure $\quad 17$

4.2. Local Structure $\quad 23$

$\begin{array}{ll}\text { References } & 27\end{array}$

\section{INTRODUCTION}

This paper describes the moduli space of slope semi-stable sheaves on a ribbon. A ribbon is a first-order thickening of a non-singular curve, and in the context of this paper, their study began with work of Bayer-Eisenbud ([BE95]) and Eisenbud-Green ([EG95]), where ribbons were used to study Green's conjecture on linear series using degeneration techniques. More recently, the first author used these techniques to study Brill-Noether loci ([Che10]). Given a linear series on a non-singular curve, the technique is to specialize the curve to a ribbon and then specialize the linear series to a series on the ribbon. Here one encounters a difficulty: It is only possible to specialize the linear series if the specialization is allowed to be a generalized linear series.

2010 Mathematics Subject Classification. Primary 14D20, Secondary 14C20.

Key words and phrases. Ribbon, Semi-stable sheaf, Generalized line bundle.

During the preparation of this work the first author was partially supported by NSF grant DMS-1200329 and NSF CAREER grant DMS-1350396. The second author was partially supported by NSF grant DMS-0502170.

1

(c) 2015. This manuscript version is made available under the Elsevier user license

http://www.elsevier.com/open-access/userlicense/1.0/ 
Recall that a linear series is a line bundle $\mathcal{I}$ together with a subspace of the space of global sections. Eisenbud and Green defined a generalized linear series on a ribbon by allowing $\mathcal{I}$ to be a more general coherent sheaf that they called a generalized line bundle. They observed that the set of all generalized line bundles of fixed degree cannot be parameterized by an algebraic $k$-scheme because the class of such sheaves is unbounded. Given a finite birational morphism $f: X^{\prime} \rightarrow X$ of ribbons, the direct image $\mathcal{I}:=f_{*}\left(\mathcal{I}^{\prime}\right)$ of a line bundle $\mathcal{I}^{\prime}$ is a generalized line bundle, and we obtain all generalized line bundles on $X$ by varying over all possible $X^{\prime}$. The genus of $X^{\prime}$ is an important invariant of $\mathcal{I}=f_{*}\left(\mathcal{I}^{\prime}\right)$, so following Drézet ([Dré08, §5.4]), we define the index $b(\mathcal{I})$ of $\mathcal{I}$ by $b(\mathcal{I}):=g(X)-g\left(X^{\prime}\right)$. The index of a generalized line bundle can be arbitrarily negative, and unboundedness follows. The question posed by Eisenbud and Green ([EG95, p. 758, midpage]) is: If $X$ is a rational ribbon (i.e. $X_{\text {red }} \cong \mathbb{P}^{1}$ ), then is it possible to compactify the moduli space of degree 0 line bundles on $X$ by a moduli space of degree 0 generalized line bundles with non-negative index?

Requiring that the index is non-negative is one way to recover boundedness. Another way is to impose the condition of slope semi-stability. In the general theory of moduli of sheaves, it has long been recognized that many natural classes of sheaves are unbounded, but one can recover boundedness by considering sheaves that satisfy slope semi-stability. In great generality, Simpson ([Sim94]) has constructed moduli spaces $\mathrm{M}\left(\mathcal{O}_{X}, P\right)$ of semi-stable sheaves with Hilbert polynomial $P$ on a polarized scheme $(X, \mathcal{L}) \cdot \operatorname{M}\left(\mathcal{O}_{X}, P\right)$ is a coarse moduli space of semi-stable sheaves, but sheaves up to $G r$-(or Jordan) equivalence rather than isomorphism. Sheaves satisfying the stronger condition of stability sweep out an open locus $\mathrm{M}_{\mathrm{s}}\left(\mathcal{O}_{X}, P\right) \subset \mathrm{M}\left(\mathcal{O}_{X}, P\right)$. For stable sheaves, Gr-equivalence coincides with isomorphism, and $\mathrm{M}_{\mathrm{s}}\left(\mathcal{O}_{X}, P\right)$ is the fine moduli space of stable sheaves. The goal of this paper is to describe the Simpson moduli spaces $\mathrm{M}\left(\mathcal{O}_{X}, P\right)$ and $\mathrm{M}_{\mathrm{s}}\left(\mathcal{O}_{X}, P\right)$ when $X$ is a ribbon.

Throughout the paper, we denote by $g$ the genus of a ribbon $X$ and by $\bar{g}$ the genus of its reduced support $X_{\text {red }}$. Given a polarized ribbon $(X, \mathcal{L})$, we study the Simpson moduli space parameterizing semi-stable sheaves with Hilbert polynomial $P_{d}(t):=\operatorname{deg}(\mathcal{L}) t+d+1-g$, the Hilbert polynomial of a degree $d$ line bundle (see Section 3 for more on $P_{d}$ ).

There are two types of sheaves that are parameterized by $\mathrm{M}\left(\mathcal{O}_{X}, P_{d}\right)$ : generalized line bundles on $X$ and rank 2 vector bundles on $X_{\text {red }}$. More precisely, we have

Theorem A. Let $(X, \mathcal{L})$ be a polarized ribbon. If $\mathcal{F}$ is a coherent sheaf on $X$, then $\mathcal{F}$ corresponds to a point of $\mathrm{M}\left(\mathcal{O}_{X}, P_{d}\right)$ (resp. $\mathrm{M}_{\mathrm{s}}\left(\mathcal{O}_{X}, P_{d}\right)$ ) if and only if $\mathcal{F}$ is isomorphic to one of the following sheaves:

- a degree d generalized line bundle $\mathcal{I}$ of index less than or equal to (resp. strictly less than) $1+g-2 \bar{g}$; 
- the direct image $i_{*} \mathcal{E}$ of a rank 2 , slope semi-stable (resp. slope stable) vector bundle on $X_{\text {red }}$ of degree $d+2 \bar{g}-1-g$. Here $i: X_{\text {red }} \hookrightarrow X$ is the inclusion map.

This is a reformulation of Theorem 3.5. When $X_{\text {red }}=\mathbb{P}^{1}$, this theorem was proven by Yang [Yan03, Thm. 5.8], and there is also significant overlap between Theorem A and earlier work of Drézet and Donagi-Ein-Lazarsfeld. The relation between their work and the present paper is discussed at the end of this section.

Consider Theorem A when $X$ is a rational ribbon (i.e. $\bar{g}=0$ ), which was the case considered in [BE95]. The theorem then asserts that the stable locus $\mathrm{M}_{\mathrm{S}}\left(\mathcal{O}_{X}, P_{0}\right)$ of the Simpson moduli space is the fine moduli space parameterizing generalized line bundles of index at most $g$. When $g$ is even, every semi-stable sheaf is stable, and $\mathrm{M}_{\mathrm{s}}\left(\mathcal{O}_{X}, P_{0}\right)$ is projective. Otherwise, $\mathrm{M}_{\mathrm{S}}\left(\mathcal{O}_{X}, P_{0}\right)$ is not projective, and its complement in the projective scheme $\mathrm{M}\left(\mathcal{O}_{X}, P_{0}\right)$ consists of a single point. In other words, the Simpson moduli space satisfies the properties Eisenbud and Green ask for precisely when $g$ is even. This result is restated as Corollary 4.1 in Section 4.

We prove several results about the geometry of $\mathrm{M}\left(\mathcal{O}_{X}, P_{d}\right)$. The following theorem enumerates the irreducible components of $\mathrm{M}\left(\mathcal{O}_{X}, P_{d}\right)$.

Theorem B. Let $X$ be a ribbon. Set

$$
n:= \begin{cases}\lfloor(g+2) / 2\rfloor-\bar{g} & \text { if d is even } \\ \lfloor(g+1) / 2\rfloor-\bar{g} & \text { if } d \text { is odd } .\end{cases}
$$

Assume there exists a stable generalized line bundle of degree d (i.e. $g>$ $2 \bar{g}-1$ holds $)$. Then $\mathrm{M}\left(\mathcal{O}_{X}, P_{d}\right)$ has exactly $n$ irreducible components of dimension $g$ whose general element corresponds to a generalized line bundle.

There is at most one additional component. When it exists, this component is of dimension $4 \bar{g}-3$ and the general element corresponds to a stable rank 2 vector bundle on $X_{\text {red. }}$. This additional component does not exist when $\bar{g}=0,1$ but does exist when the two conditions $\bar{g} \geq 2$ and $4 \bar{g}-3 \geq g$ are satisfied.

This is a combination of Theorems 4.6 and 4.7. We remark that for $\bar{g} \geq 2$ and $4 \bar{g}-3<g$, we do not know whether $\mathrm{M}\left(\mathcal{O}_{X}, P_{d}\right)$ has an irreducible component whose general element is a stable rank 2 vector bundle (see Question 4.8).

As with Theorem A, when $X_{\text {red }}=\mathbb{P}^{1}$, Theorem B was proven by Yang [Yan03, Thm. 5.8], and we discuss the relation between this result and results in the literature at the end of this introduction.

We also verify connectedness of $\mathrm{M}\left(\mathcal{O}_{X}, P_{d}\right)$. The statement below is a restatement of Theorem 4.10.

Theorem C. For a ribbon $X$, the moduli space $\mathrm{M}\left(\mathcal{O}_{X}, P_{d}\right)$ is connected.

Finally, we determine the smooth locus of $\mathrm{M}\left(\mathcal{O}_{X}, P_{d}\right)$. 
Theorem D. Let $X$ be a ribbon. If $\bar{g} \geq 2$ and $g \geq 4 \bar{g}-2$, then the smooth locus of $\mathrm{M}\left(\mathcal{O}_{X}, P_{d}\right)$ is equal to the open subset of line bundles on $X$.

This is Corollary 4.14, which is a consequence of the computation of the tangent space to $\mathrm{M}_{\mathrm{S}}\left(\mathcal{O}_{X}, P_{d}\right)$ at a point (Proposition 4.11), and these tangent space computations were established earlier in work by Inaba and Drézet that we discuss at the end of this introduction. When $\bar{g}=0,1$ or $g<\bar{g}-2$, the techniques we use to prove Thereom $\mathrm{D}$ do not allow us to compute the smooth locus. It would be interesting to extend the theorem to these cases, and we discuss this topic in more detail after the proof of Corollary 4.14 .

The results of this paper relate to earlier work of the second author. In [Kas13], he related the line bundle locus in family of fine moduli spaces of sheaves to the Néron model. The main result ([Kas13, Thm. 3.10]) states that given a family of curves over a Dedekind scheme $B$ with regular total space $X_{b}$, the line bundle locus in a suitable family of fine Simpson moduli spaces of sheaves is the Néron model of its generic fiber. However, for some families, a suitable Simpson family may not exist.

Indeed, the existence of such a Simpson family implies that Raynaud's maximal separated quotient of the Picard functor is isomorphic to the Néron model of its generic fiber, and these two schemes are not isomorphic for certain families of curves constructed by Raynaud, Liu, and Lorenzini ([Ray70, 9.2], [LLR04, 9.2]). Their construction is arithmetic. They work over the spectrum of a mixed characteristic DVR with inseparable residue field, and using a purely inseparable element of the residue field, their family is an explicit family of plane curves. The main results of this paper explain the geometry of this example. In the examples from [Ray70] and [LLR04], the geometric special fiber $X$ is non-reduced, as it must be by [Ray70, Thm. 8.1.4]. Theorem A provides a direct, geometric explanation for why a suitable Simpson family does not exist: For certain non-reduced $X$, e.g. rational ribbons of odd genus, the Simpson moduli space always contains a strictly semi-stable point.

Now we compare our results to past work on ribbons. In [Ina04], MichiAki Inaba studied the moduli space of stable sheaves on a non-reduced scheme and, in particular, proved results about the local structure of the moduli space of slope stable sheaves on a ribbon ([Ina04, Thm. 2.6]). Closest to our results is [Ina04, Rmk. 2.7], which contains tangent space computations similar to Lemma 4.13.

Beginning with [Dré06], Jean-Marc Drézet has written several papers ([Dré06], [Dré08], [Dré09], [Dré11]) studying slope semi-stable sheaves on a multiple curve, the $n$-th order analogue of a ribbon. Much of this work focuses on "quasi-locally free sheaves," a class of sheaves that includes line bundles, but not generalized line bundles. Most relevant to this paper is [Dré11] (esp. Thm. 5.4.2), which the authors became aware of while preparing the current document. In that paper, Drézet provides sufficient conditions for a 
pure sheaf of dimension 1 (there called a "torsion-free sheaf") on a primitive multiple curve to be (semi-)stable. Also relevant are Drézet's classification of pure sheaves with Hilbert polynomial $P_{d}([$ Dré08, §5.4]) and infinitesimal computations appearing in [Dré06] (especially [Dré06, Prop. 8.1.3], which includes cases of Lemma 4.12).

Finally, after this paper was written, the authors were made aware of the papers [DEL97] and [Yan03]. In [DEL97], the authors study the Hitchin and Mukai systems, and the moduli space of stable sheaves associated to a ribbon lying on a $K 3$-surface arises as the nilpotent cone of the Mukai space. The structure of the nilpotent cone is studied in some detail. In particular, under the additional hypothesis that the nilradical $\mathcal{N}$ of $X$ is isomorphic to the anti-canonical bundle $K_{X_{\text {red }}}^{-1}$ of $X_{\text {red }}$ (so $g=4 \bar{g}-3$ ), Theorem B is stated as [DEL97, Thm. 3.2].

In [Yan03], the author studies the moduli space of sheaves on a rational ribbon, and the main result [Yan03, Thm. 5.8] is our Theorems A and B in the special case $X$ is a rational ribbon. The methods used in [Yan03] are different from the methods used in this paper: Yang studies sheaves on a ribbon by considering them as extensions of sheaves on the reduced subcurve $X_{\text {red}}$.

This paper is organized as follows. In Section 2, we collect some basic definitions and facts about coherent sheaves on ribbons that are used later. Theorem A is proven in Section 3, as a culmination of results on semistability. Finally, the geometry of $\mathrm{M}\left(\mathcal{O}_{X}, P_{d}\right)$ is studied in Section 4 . The main results proven in that section are Theorems $\mathrm{B}, \mathrm{C}$ and $\mathrm{D}$.

Acknowledgements. The authors would like to thank Robert Lazarsfeld for informing them of his paper [DEL97] with Donagi and Ein and for providing helpful expository suggestions. The authors would also like to thank Daniel Erman for expository feedback, Yusuf Mustopa for enlightening conversations about vector bundles on curves, Matt Satriano for helpful discussions on homological algebra. Finally the authors thank the anonymous referee for many useful comments that helped improve the exposition of the paper.

\section{Coherent Sheaves on a RibBon}

Let us first fix some conventions. We work over an algebraically closed field $k$. A curve is an irreducible, projective $k$-scheme of dimension 1. A nonsingular curve is a curve that is smooth over $k$. A ribbon $X$ is a curve such that the reduced subscheme $X_{\text {red }}$ is a non-singular curve and the nilradical $\mathcal{N}$ is locally generated by a non-zero, square-zero element of $\mathcal{O}_{X}$. A ribbon $X$ is a rational ribbon if $X_{\text {red }}$ is isomorphic to $\mathbb{P}^{1}$.

Here we collect the facts about coherent sheaves on ribbons that are needed to describe the Simpson moduli space. In this section, let $X$ be a fixed ribbon with generic point $\eta$. The degree $\operatorname{deg}(\mathcal{I})$ of a coherent sheaf $\mathcal{I}$ 
on $X$ is

$$
\operatorname{deg}(\mathcal{I}):=\chi(\mathcal{I})-\chi\left(\mathcal{O}_{X}\right)
$$

The genus $g(X)$ of a curve $X$ is

$$
g:=1-\chi\left(\mathcal{O}_{X}\right)
$$

By definition, the nilradical $\mathcal{N}$ is locally generated by a single square-zero, but non-zero element of $\mathcal{O}_{X}$, hence it may be considered as a line bundle on $X_{\text {red }}$. Using the exact sequence

$$
\mathcal{N} \hookrightarrow \mathcal{O}_{X} \rightarrow \mathcal{O}_{X_{\text {red }}}
$$

the degree of $\mathcal{N}$ as a line bundle on $X_{\text {red }}$ can be computed as

$$
\operatorname{deg}(\mathcal{N})=2 \bar{g}-1-g,
$$

where $g$ is the genus of $X$ and $\bar{g}$ the genus of $X_{\text {red }}$. This quantity plays an important role in many of the results of this paper, appearing, for example, as a bound in Theorem A.

Notice that we have defined the genus $g$ by $g:=1-\chi\left(\mathcal{O}_{X}\right)$. The genus of an integral curve $X$ always equals $h^{1}\left(X, \mathcal{O}_{X}\right)$, but this equality may fail to hold when $X$ is a ribbon. Indeed, the two numbers are equal precisely when $\mathcal{N}$ has no global sections, which holds when $g$ is sufficiently large but not in general.

We now begin our review of coherent sheaves on a ribbon. First, some definitions from [EG95].

Definition 2.1. If $\mathcal{F}$ is a coherent sheaf on $X$, then we write $d(\mathcal{F})$ for the dimension of the support of $\mathcal{F}$. We say that $\mathcal{F}$ is pure if $d(\mathcal{F})=d(\mathcal{G})$ for all non-zero subsheaves $\mathcal{G} \subset \mathcal{F}$.

If $\mathcal{F}$ is a coherent sheaf, then we say that a regular function $f \in H^{0}\left(U, \mathcal{O}_{X}\right)$ is a non-zero divisor on $\mathcal{F}$ if the multiplication map $f \cdot_{-}:\left.\left.\mathcal{F}\right|_{U} \rightarrow \mathcal{F}\right|_{U}$ is injective. The sheaf $\mathcal{F}$ is said to be torsion-free if every non-zero divisor on $\mathcal{O}_{X}$ is a non-zero divisor on $\mathcal{F}$. The sheaf $\mathcal{F}$ is said to be rank 1 if the generic stalk $\mathcal{F}_{\eta}$ is isomorphic to $\mathcal{O}_{X, \eta}$. A generalized line bundle is a coherent sheaf that is rank 1 and torsion-free.

Next, we describe some basic properties of pure sheaves on a ribbon. Some of them are well-known to experts, but we include their proofs for the reader's convenience.

Lemma 2.2. Let $\mathcal{I}$ be a coherent sheaf on a ribbon $X$ satisfying $d(\mathcal{I})=1$. Then $\mathcal{I}$ is torsion-free if and only if $\mathcal{I}$ is pure.

The following concise proof was suggested to the authors by the referee.

Proof. By definition, $\mathcal{I}$ is not torsion-free $\Longleftrightarrow$ there exists an open affine subset $U$, a non-zero divisor $f \in H^{0}\left(U, \mathcal{O}_{X}\right)$, and a non-zero $g \in H^{0}(U, \mathcal{I})$ such that $f g=0 \Longleftrightarrow$ there exists a non-zero $g \in H^{0}(U, \mathcal{I})$ such that $\left.\operatorname{ann}(g) \not \subset \mathcal{N}\right|_{U} \Longleftrightarrow$ there exists a non-zero $g \in H^{0}(U, \mathcal{I})$ with finite support $\Longleftrightarrow \mathcal{I}$ has a non-zero subsheaf with finite support $\Longleftrightarrow \mathcal{I}$ is not pure. 
Lemma 2.3. If $\mathcal{I}$ is a pure sheaf on a ribbon, then $\underline{\operatorname{End}}(\mathcal{I})$ is pure.

Proof. If $d(\mathcal{I})=0$, then $d(\underline{\operatorname{End}}(\mathcal{I}))=0$, hence $\underline{\operatorname{End}}(\mathcal{I})$ is pure. Suppose that $d(\mathcal{I})=1$. It is enough to prove that if $\phi$ is an element of the stalk of $\underline{\operatorname{End}}(\mathcal{I})$ at the closed point $x$ that is annihilated by the maximal ideal, then $\phi=0$. Given such a $\phi$, for every $s \in \mathcal{I}_{x}$ the element $\phi(s) \in \mathcal{I}_{x}$ is annihilated by the maximal ideal, so $\phi(s)=0$ as $\mathcal{I}$ is pure.

Lemma 2.4. If $\mathcal{I}$ is a pure sheaf of generic length 2 , then the kernel of the map $\varphi: \mathcal{O}_{X} \rightarrow \underline{\operatorname{End}}(\mathcal{I})$ is either the zero ideal $(0)$ or the nilradical $\mathcal{N}$.

The following argument was suggested by the referee.

Proof. Set $\mathcal{K}$ equal to the kernel of $\varphi$. The quotient $\mathcal{O}_{X} / \mathcal{K}$ injects into $\underline{\operatorname{End}}(\mathcal{I})$, and the quotient is nonzero because e.g. the constants $k \subset \mathcal{O}_{X}$ map to nonzero endomorphisms. Since $\operatorname{End}(\mathcal{I})$ is pure (by the previous lemma), we can conclude that $\mathcal{O}_{X} / \mathcal{K}$ is pure of dimension 1 . By primary decomposition, $\mathcal{K}$ must be contained in the nilradical (as the primes associated to $\mathcal{K}$ must all be height 0 and $\mathcal{N}$ is the only prime with this property).

The localization $\mathcal{N}_{\eta}$ of the nilradical at the generic point $\eta \in X$ is free of rank 1 over the field $\mathcal{O}_{X_{\text {red }}, \eta}$, so the localization $\mathcal{K}_{\eta}$ of the kernel is either zero or all of $\mathcal{N}_{\eta}$. If $\mathcal{K}_{\eta}=0$, then $\mathcal{K}=0$ by purity. Otherwise the quotient $\mathcal{N} / \mathcal{K}$ has the property that the localization at $\eta$ is zero, hence the quotient is supported on a finite set of point. But the quotient is a submodule of the pure module $\mathcal{O}_{X} / \mathcal{K}$, so $\mathcal{N} / \mathcal{K}=0$ and hence $\mathcal{K}=\mathcal{N}$.

One application of Lemma 2.2 is the following result, which provides an alternative characterization of generalized line bundles.

Lemma 2.5. Let $X$ be a ribbon and $\mathcal{I}$ a pure sheaf whose generic stalk is an $\mathcal{O}_{X, \eta}$-module of length 2 . Then $\mathcal{I}$ is a generalized line bundle if and only if the map $\varphi: \mathcal{O}_{X} \rightarrow \underline{\operatorname{End}}(\mathcal{I})$ is injective.

Proof. Let $X$ and $\mathcal{I}$ be given. We have shown that torsion-free is equivalent to pure (Lemma 2.2), so the content of the lemma is that $\mathcal{I}$ is rank 1 if and only if $\varphi$ is injective. The map $\varphi$ factors as

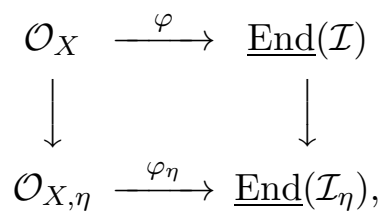

where $\eta$ is the generic point.

When $\mathcal{I}$ is a generalized line bundle, the vertical maps are injective (because $\mathcal{O}_{X}$ and $\mathcal{I}$ are pure) and $\mathcal{O}_{X, \eta} \rightarrow \underline{\text { End }}\left(\mathcal{I}_{\eta}\right)$ is an isomorphism (because $\mathcal{I}$ is rank 1$)$. We conclude that $\varphi$ is injective.

Conversely, assume that $\varphi$ is injective. Pick a generator $\epsilon$ of the generic stalk $\mathcal{N}_{\eta}$. By hypothesis, multiplication by $\epsilon$ on $\mathcal{I}_{\eta}$ is not the zero map, so there exists an element $s_{0} \in \mathcal{I}_{\eta}$ with $\epsilon s_{0} \neq 0$. We claim that the map $\varphi_{\eta}$ is an 
isomorphism. The kernel of $\varphi_{\eta}$ is $\operatorname{ann}\left(s_{0}\right)$, which is contained in $\mathcal{N}_{\eta}$ (because $\mathcal{I}$ is pure). In fact, this containment is proper because $\epsilon s_{0} \neq 0$. Since $\mathcal{N}_{\eta}$ is an $\mathcal{O}_{X_{\text {red }}, \eta}$-module and $\mathcal{O}_{X_{\text {red }}, \eta}$ is a field, we conclude that ann $\left(s_{0}\right)=0$, proving injectivity. Surjectivity also follows: Both the module $\mathcal{I}_{\eta}$ and the submodule generated by $s_{0}$ have length 2 , so they must coincide. We conclude that $\mathcal{I}_{\eta}$ is isomorphic to $\mathcal{O}_{X, \eta}$, and the proof is complete.

The following result provides a classification of pure sheaves whose generic stalk has length 2. It can be found in the work of Drézet, but we include a proof for the sake of completeness.

Proposition 2.6 ([Dré06, §5.4]). Let $X$ be a ribbon and $\mathcal{I}$ a pure sheaf whose generic stalk is an $\mathcal{O}_{X, \eta}$-module of length 2 . Then $\mathcal{I}$ is isomorphic to one of the following:

(1) a generalized line bundle;

(2) the direct image $i_{*}(\mathcal{E})$ of a rank 2 vector bundle $\mathcal{E}$ on $X_{\text {red }}$ under the inclusion map $i: X_{\text {red }} \hookrightarrow X$.

Proof. Given $\mathcal{I}$, consider the natural map $\mathcal{O}_{X} \rightarrow \underline{\operatorname{End}}(\mathcal{I})$. By Lemma 2.4, the kernel of this map is either 0 or the nilradical $\mathcal{N}$. When the kernel is 0 , Lemma 2.5 states that $\mathcal{I}$ is a generalized line bundle. Thus, we focus on the case where the kernel is $\mathcal{N}$.

In this case, consider $\mathcal{I}$ as a module $\mathcal{E}$ over $\mathcal{O}_{X} / \mathcal{N}=\mathcal{O}_{X_{\text {red }}}$. Certainly, $\mathcal{E}$ then satisfies $\mathcal{I}=i_{*}(\mathcal{E})$. The module $\mathcal{E}$ is pure on the smooth curve $X_{\text {red }}$, hence locally free and the generic rank is 2 by hypothesis. In other words, $\mathcal{E}$ is a rank 2 vector bundle on $X_{\text {red }}$, completing the proof.

We conclude this section with a discussion of the sheaves appearing in Proposition 2.6. At this level of generality, there is not much to be said about the classification of rank 2 vector bundles on $X_{\text {red }}$. When $X_{\text {red }}$ has genus 0 , every vector bundle is a direct sum of line bundles. But in general, there are non-split rank 2 vector bundles and the classification is more complicated. In Section 3, we introduce stability conditions and the semi-stable vector bundles on $X_{\text {red }}$ are coarsely parameterized by the corresponding Simpson moduli space.

There is a classification of generalized line bundles on $X$, which can be found in [EG95, Thm. 1.1]. Given an effective Cartier divisor $D$ on $X_{\text {red }}$, we can consider $D$ as a closed subscheme of $X$ and form the blow-up $f: X^{\prime}:=$ $\mathrm{Bl}_{D}(X) \rightarrow X$. The blow-up $X^{\prime}$ is itself a ribbon and $f$ is a finite morphism. In fact, the quotient $f_{*}\left(\mathcal{O}_{X^{\prime}}\right) / \mathcal{O}_{X}$ is isomorphic to $\mathcal{O}_{D}$. If $\mathcal{L}^{\prime}$ is a line bundle on $X^{\prime}$, then the direct image $\mathcal{I}:=f_{*}\left(\mathcal{L}^{\prime}\right)$ is a generalized line bundle. [EG95, Thm. 1.1] states that every generalized line bundle is of this form for a unique blow-up $f: X^{\prime} \rightarrow X$ and a unique line bundle $\mathcal{L}^{\prime}$. Given $\mathcal{I}$, we call $f: X^{\prime} \rightarrow X$ the associated blow-up. Following Drézet ([Dré08, §5.4], we use the Green-Eisenbud result to define an invariant of $\mathcal{I}$.

Definition 2.7. Let $\mathcal{I}=f_{*}\left(\mathcal{I}^{\prime}\right)$ be a generalized line bundle on $X$ for the blow-up $f: X^{\prime}=\mathrm{Bl}_{D}(X) \rightarrow X$ and the line bundle $\mathcal{I}^{\prime}$ on $X^{\prime}$. As a divisor 
on $X_{\text {red }}$, write $D=\sum n_{p} p$. Given a point $p_{0} \in X$, we define the local index of $\mathcal{I}$ at $p_{0}$, written $b_{p_{0}}(\mathcal{I})$, to be $b_{p_{0}}(\mathcal{I}):=n_{p_{0}}$. The local index sequence is the collection $\left\{b_{p_{0}}(\mathcal{I}): b_{p_{0}}(\mathcal{I}) \neq 0\right\}$, and the index of $\mathcal{I}$ is defined by $b(\mathcal{I}):=\sum b_{p}(\mathcal{I})$. In particular, we have

$$
b(\mathcal{I})=g-g^{\prime}
$$

which follows from $f_{*}\left(\mathcal{O}_{X^{\prime}}\right) / \mathcal{O}_{X} \cong \mathcal{O}_{D}$ and $b(\mathcal{I})=\operatorname{deg}(D)$.

The integers $b(\mathcal{I})$ and $b_{p_{0}}(\mathcal{I})$ can also be defined intrinsically. The index $b(\mathcal{I})$ is the length of $\operatorname{End}(\mathcal{I}) / \mathcal{O}_{X}$, while $b_{p_{0}}(\mathcal{I})$ is the length of the localization $\underline{\text { End }}\left(\mathcal{I}_{p_{0}}\right) / \mathcal{O}_{X, p_{0}}$. Later in Fact 2.15 we show that if $\mathcal{I}=\mathcal{I}_{p_{0}}$ is the ideal of a closed point $p_{0} \in X$, then $\mathcal{I}$ is a generalized line bundle of degree -1 with local index at $p_{0}$ equal to 1.

Later on we will use the following basic relation between the degree and local index of a generalized line bundle ([EG95, p. 759, midpage]).

Fact 2.8. If $\mathcal{I}$ is a generalized line bundle, then $\operatorname{deg}(\mathcal{I})-b(\mathcal{I})=2 b\left(\mathcal{I}^{\prime}\right)$ is even.

One consequence of the classification theorem of Green-Eisenbud is that the Jacobian $J^{0}(X)$ (i.e. the space of degree 0 line bundles) acts transitively on the set of generalized line bundles with fixed local index sequence. This fact will be used later, so we record it.

Lemma 2.9. Let $X$ be a ribbon. If $\mathcal{I}_{1}$ and $\mathcal{I}_{2}$ are two generalized line bundles that have the same local index at $p$ for all $p \in X$, then there exists a line bundle $\mathcal{L}$ on $X$ such that $\mathcal{I}_{1}$ is isomorphic to $\mathcal{L} \otimes \mathcal{I}_{2}$. Furthermore, any two line bundles with this property differ by an element of $\operatorname{ker}\left(f^{*}: \operatorname{Pic}(X) \rightarrow\right.$ $\left.\operatorname{Pic}\left(X^{\prime}\right)\right)$, where $f: X^{\prime} \rightarrow X$ is the blow-up associated to $\mathcal{I}_{i}$.

Proof. If $f: X^{\prime} \rightarrow X$ is the blow-up as in Definition 2.7, then we can write $\mathcal{I}_{i}=f_{*}\left(\mathcal{L}_{i}\right)$ for line bundles $\mathcal{L}_{1}$ and $\mathcal{L}_{2}$ on $X^{\prime}$. Since $f_{*}\left(\mathcal{O}_{X^{\prime}}^{*}\right) / \mathcal{O}_{X}^{*}$ has finite support, the map $H^{1}\left(X, \mathcal{O}_{X}^{*}\right) \rightarrow H^{1}\left(X^{\prime}, \mathcal{O}_{X^{\prime}}^{*}\right)$ is surjective, i.e., the map $f^{*}: \operatorname{Pic}(X) \rightarrow \operatorname{Pic}\left(X^{\prime}\right)$ is surjective. Therefore, we can find a line bundle $\mathcal{L}$ such that $f^{*}(\mathcal{L})=\mathcal{L}_{1} \otimes \mathcal{L}_{2}^{-1}$. An application of the projection formula shows that this line bundle satisfies the desired conditions, and that any other line bundle $\mathcal{M}$ with this property must satisfy $f^{*}(\mathcal{M}) \cong f^{*}(\mathcal{L})$.

The structure of the Jacobian $J^{0}(X)$ can be described explicitly: The pullback map $i^{*}: J^{0}(X) \rightarrow J^{0}\left(X_{\text {red }}\right)$ from the Jacobian of $X$ to the Jacobian of $X_{\text {red }}$ is surjective with kernel equal to the vector space group associated to $H^{1}\left(X_{\text {red }}, \mathcal{N}\right)$. This description of $J^{0}(X)$ can be found in [Dré08, $\S 2.3$ ] or [BLR90, Sect. 9.2]. One approach is to use the description of $J^{0}(X)$ as the identity component of the moduli space $\operatorname{Pic}(X)$ of line bundles on $X$. In [BLR90], $\operatorname{Pic}(X)$ is described as the scheme that represents the étale sheaf $R^{1} g_{*}\left(\mathcal{O}_{X}^{*}\right)$, where $g: X \rightarrow \operatorname{Spec}(k)$ is the structure morphism, and then computed using long exact sequence associated to the "exponential sequence"

$$
\mathcal{N} \hookrightarrow \mathcal{O}_{X}^{*} \rightarrow \mathcal{O}_{X_{\text {red }}}^{*}
$$


We collect the following result which will be used in Section 4 .

Fact 2.10. Let $X$ be a ribbon.

(1) The dimension of $J^{0}(X)$ equals $h^{1}\left(X, \mathcal{O}_{X}\right)$;

(2) For $g \geq \bar{g}+1, J^{0}(X)$ is not a proper $k$-scheme (as then $H^{1}\left(X_{\text {red }}, \mathcal{N}\right) \neq$ $0)$.

If we pass from $X$ to its completed local ring at a point, then we can describe generalized line bundles more explicitly.

Definition 2.11. Set $\mathcal{O}_{0}:=k[[s, \epsilon]] /\left(\epsilon^{2}\right)$. Define the $n$-th blow-up algebra to be $\mathcal{O}_{n}:=k[[\tilde{s}, \tilde{\epsilon}]] /\left(\tilde{\epsilon}^{2}\right)$, considered as an $\mathcal{O}_{0}$-algebra via $s \mapsto \tilde{s}, \epsilon \mapsto \tilde{\epsilon} \tilde{s}^{n}$. We write $I_{n}$ for $\mathcal{O}_{n}$, considered as an $\mathcal{O}_{0}$-module.

The following lemma justifies the term "blow-up algebra."

Lemma 2.12. The blow-up of $\operatorname{Spec}\left(\mathcal{O}_{0}\right)$ along the ideal $\left(s^{n}, \epsilon\right)$ is $\operatorname{Spec}\left(\mathcal{O}_{n}\right) \rightarrow$ $\operatorname{Spec}\left(\mathcal{O}_{0}\right)$.

Proof. By definition, the blow-up is the projectivization of the Rees algebra

$$
R_{+}:=\mathcal{O}_{0} \bigoplus\left(s^{n}, \epsilon\right) T \bigoplus\left(s^{2 n}, \epsilon s^{n}\right) T^{2} \bigoplus \cdots=\bigoplus_{k=0}^{\infty}\left(s^{n}, \epsilon\right)^{k} T^{k} .
$$

Here $T$ is a formal variable and $R_{+}$is graded so that $T$ has grade 1 and elements of $\mathcal{O}_{0}$ grade 0 . As an $\mathcal{O}_{0}$-algebra, $R_{+}$is generated by $U:=s^{n} T$ and $V:=\epsilon T$. If $\left(R_{+}\left[U^{-1}\right]\right)_{0}$ is the grade 0 subring of the localization $R\left[U^{-1}\right]$ and similarly with $\left(R_{+}\left[V^{-1}\right]\right)_{0}$, then by definition, Proj $R_{+}$is covered by the open affine schemes $\operatorname{Spec}\left(\left(R_{+}\left[U^{-1}\right]\right)_{0}\right)$ and $\operatorname{Spec}\left(\left(R_{+}\left[V^{-1}\right]\right)_{0}\right)$.

The ring $R_{+}\left[V^{-1}\right]$, and thus $\left(R_{+}\left[V^{-1}\right]\right)_{0}$, is zero because $V$ is both invertible and a zero divisor. As a consequence, the blow-up is equal to $\operatorname{Spec}\left(\left(R_{+}\left[U^{-1}\right]\right)_{0}\right)$, and we complete the proof by showing this algebra is isomorphic to $\mathcal{O}_{n}$.

In $\left(R_{+}\left[U^{-1}\right]\right)_{0}$, we have

$$
\begin{aligned}
s^{n} V / U & =\epsilon U / U \\
& =\epsilon .
\end{aligned}
$$

This shows that the rules $\tilde{s} \mapsto s, \tilde{\epsilon} \mapsto V / U$ define a $\mathcal{O}_{0}$-algebra homomorphism $\mathcal{O}_{n} \rightarrow\left(R_{+}\left[U^{-1}\right]\right)_{0}$. This homomorphism is surjective because $V / U$ and $1=U / U$ generate $\left(R_{+}\left[U^{-1}\right]\right)_{0}$ as a $\mathcal{O}_{0}$-algebra.

To show injectivity, let $f(\tilde{s})+\epsilon g(\tilde{s}) \in \mathcal{O}_{n}$, with $f(\tilde{s}), g(\tilde{s}) \in k[[s]]$, be an element that maps to zero. By the definition of localization, we must have

$$
U^{b}(U f(s)+V g(s))=0 \text { in } R_{+}
$$

or equivalently

$$
s^{n b}\left(s^{n} f(s)+\epsilon g(s)\right)=0 \text { in }\left(s^{n}, \epsilon\right)^{b+1} \subset \mathcal{O}_{0} .
$$

Since $s^{n b}$ is a nonzero divisor in $\mathcal{O}_{0}$ and $s^{n}$ a nonzero divisor in $\mathcal{O}_{n}$, we can conclude that $f(\tilde{s})+\tilde{\epsilon} g(\tilde{s})=0$. 
The formation of a blow-up commutes with passing to the completed local ring, so if $f: X^{\prime}:=\mathrm{Bl}_{D}(X) \rightarrow X$ is the blow-up from Definition 2.7 and $q \in X^{\prime}$ a point mapping to $p \in X$, then the induced map $\widehat{\mathcal{O}}_{X, p} \rightarrow$ $\widehat{\mathcal{O}}_{X^{\prime}, q}$ is isomorphic to $\mathcal{O}_{0} \rightarrow \mathcal{O}_{n_{p}}$. Combining this observation with [EG95, Thm. 1.1], we deduce the following lemma.

Lemma 2.13. Let $X$ be a ribbon, $p_{0} \in X$ a point and $\mathcal{I}$ a generalized line bundle. Fix an isomorphism between $\mathcal{O}_{0}$ and $\widehat{\mathcal{O}}_{X, p_{0}}$. Then under this isomorphism, $\mathcal{I} \otimes \widehat{\mathcal{O}}_{X, p_{0}}$ is identified with a module isomorphic to $I_{n}$, where $n=b_{p_{0}}(\mathcal{I})$.

Proof. Write $\mathcal{I}=f_{*}\left(\mathcal{L}^{\prime}\right)$ for the blow-up $f: X^{\prime}:=\mathrm{Bl}_{D}(X) \rightarrow X$ and a line bundle $\mathcal{L}^{\prime}$ on $X^{\prime}$. If $q \in X^{\prime}$ is the unique point mapping to $p$, then the identification of $\widehat{\mathcal{O}}_{X, p}$ with $\mathcal{O}_{0}$ extends to an identification of the map $\hat{f}: \widehat{\mathcal{O}}_{X, p} \rightarrow \widehat{\mathcal{O}}_{X^{\prime}, q}$ on completed local rings with $\mathcal{O}_{0} \rightarrow \mathcal{O}_{n}$. In particular, $\mathcal{I} \otimes \widehat{\mathcal{O}}_{X, p}$ is identified with the direct image of a line bundle on $\mathcal{O}_{n}$, and such a module is isomorphic to $\mathcal{O}_{n}$ itself.

One may easily compute that $\mathcal{O}_{0} \rightarrow \mathcal{O}_{n}$ is the algebra extension corresponding to the blow-up of $\mathcal{O}_{0}$ along $\left(s^{n}, \epsilon\right)$. Given a blow-up $f: X^{\prime}:=$ $\mathrm{Bl}_{D}(X) \rightarrow X$ as in Definition 2.7 and a point $q \in X^{\prime}$ mapping to $p \in X$, the induced map $\widehat{\mathcal{O}}_{X, p} \rightarrow \widehat{\mathcal{O}}_{X^{\prime}, q}$ can be identified with $\mathcal{O}_{0} \rightarrow \mathcal{O}_{n_{p}}$. Combining this observation with [EG95, Thm. 1.1], we can deduce the following lemma.

Lemma 2.14. Let $X$ be a ribbon, $p_{0} \in X$ a point and $\mathcal{I}$ a generalized line bundle. Fix an isomorphism between $\mathcal{O}_{0}$ and $\widehat{\mathcal{O}}_{X, p_{0}}$. Then under this isomorphism, $\mathcal{I} \otimes \widehat{\mathcal{O}}_{X, p_{0}}$ is identified with a module isomorphic to $I_{n}$, where $n=b_{p_{0}}(\mathcal{I})$.

Proof. Write $\mathcal{I}=f_{*}\left(\mathcal{L}^{\prime}\right)$ for the blow-up $f: X^{\prime}:=\mathrm{Bl}_{D}(X) \rightarrow X$ and a line bundle $\mathcal{L}^{\prime}$ on $X^{\prime}$. Note that $f$ is a set-theoretical bijection. If $q \in X^{\prime}$ is the unique point mapping to $p$, then the identification of $\widehat{\mathcal{O}}_{X, p}$ with $\mathcal{O}_{0}$ extends to an identification of the map $\hat{f}: \widehat{\mathcal{O}}_{X, p} \rightarrow \widehat{\mathcal{O}}_{X^{\prime}, q}$ on completed local rings with $\mathcal{O}_{0} \rightarrow \mathcal{O}_{n}$. In particular, $\mathcal{I} \otimes \widehat{\mathcal{O}}_{X, p}$ is identified with the direct image of a line bundle on $\mathcal{O}_{n}$, and such a module is isomorphic to $\mathcal{O}_{n}$ itself.

Fact 2.15. It follows from this lemma that the local index at $p_{0}$ of the ideal $\mathcal{I}_{p_{0}}$ of a closed point $p_{0}$ is 1 . Indeed, if we pass from $X$ to the ring $\mathcal{O}_{0}$, the multiplication by $s$ defines an isomorphism between the $\mathcal{O}$-module $\mathcal{O}_{1}$ and the ideal $(\epsilon, s)$.

More generally, if $\mathcal{I}$ is the ideal sheaf of the unique closed subscheme $Z$ of length $b_{0}$ that is supported at $p_{0}$ and contained in $X_{\text {red }}$ (so locally $\mathcal{I}$ is the ideal $\left(\epsilon, s^{b_{0}}\right)$ ), then $\mathcal{I}$ is a generalized line bundle of local index $b_{0}$ at $p_{0}$. The degree of this generalized line bundle is $-b_{0}$, as can be seen by computing the Euler characteristic using the exact sequence $\mathcal{I} \hookrightarrow \mathcal{O}_{X} \rightarrow \mathcal{O}_{Z}$.

For later computations, it is convenient to have an alternative description of $I_{n}$. This module can also be described as the ideal $\left(s^{n}, \epsilon\right)$ of $\mathcal{O}_{0}$. One 
isomorphism from $I_{n}$ to $\left(\epsilon, s^{n}\right)$ is given by the map sending $1 \in I_{n}$ to $s^{n}$ and $\tilde{\epsilon} \in I_{n}$ to $\epsilon$. This common module admits the following presentation:

$$
\left\langle e, f: \epsilon f=0, s^{n} f=\epsilon e\right\rangle .
$$

Here the element $e$ corresponds to $1 \in I_{n}$ and $f$ corresponds to $\tilde{\epsilon}$. This presentation in fact extends to the period resolution:

$$
\cdots \longrightarrow \mathcal{O}_{0}^{2} \stackrel{\left(\begin{array}{cc}
\epsilon & s^{n} \\
0 & -\epsilon
\end{array}\right)}{\longrightarrow} \mathcal{O}_{0}^{2} \stackrel{\left(\begin{array}{cc}
\epsilon & s^{n} \\
0 & -\epsilon
\end{array}\right)}{\longrightarrow} \mathcal{O}_{0}^{2} \longrightarrow I_{n} \longrightarrow 0 .
$$

Later, we shall use this presentation to describe how $I_{n}$ deforms.

\section{Stable Sheaves on a Ribbon}

Here we study the stability of generalized line bundles. In general, the stability of a coherent sheaf on a projective scheme is a condition defined in terms of an auxiliary ample line bundle $\mathcal{L}$. However, on a ribbon the stability condition is independent of $\mathcal{L}$, as we will see. To fix ideas, let us first work with a polarized ribbon $(X, \mathcal{L})$. As before, we write $g$ for the genus of $X, \bar{g}$ for the genus of $X_{\text {red }}$ and $\mathcal{N}$ for the nilradical of $X$. We now recall the definition of the Hilbert polynomial.

Given a coherent sheaf $\mathcal{I}$ on $X$, the Hilbert Polynomial $P(\mathcal{I}, t)$ of $\mathcal{I}$ with respect to $\mathcal{L}$ is the unique polynomial satisfying

$$
P(\mathcal{I}, n)=\chi\left(\mathcal{I} \otimes \mathcal{L}^{\otimes n}\right) \text { for all } n \in \mathbb{N} .
$$

The basic features of the Hilbert polynomial are described in e.g. [AK79, pp. 21-22]. When $\mathcal{I}$ is a generalized line bundle of degree $d$, its Hilbert polynomial is of the form

$$
\begin{aligned}
P_{d}(t) & =\operatorname{deg}(\mathcal{L}) \cdot t+\chi(\mathcal{I}) \\
& =\operatorname{deg}(\mathcal{L}) \cdot t+d+1-g .
\end{aligned}
$$

Recall that $d(\mathcal{I})$ is the dimension of the support of $\mathcal{I}$. If $d(\mathcal{I})=1$, the slope $\mu(\mathcal{I})$ of $\mathcal{I}$ with respect to $\mathcal{L}$ is defined to be

$$
\mu(\mathcal{I}):=\chi(\mathcal{I}) / \operatorname{deg}(\mathcal{L}) .
$$

We say that $\mathcal{I}$ is slope semi-stable with respect to $\mathcal{L}$ if $\mathcal{I}$ is pure and for all non-zero subsheaves $\mathcal{J} \subset \mathcal{I}$ we have

$$
\mu(\mathcal{J}) \leq \mu(\mathcal{I}) .
$$

If this inequality is always strict for $\mathcal{J} \neq \mathcal{I}$, we say $\mathcal{I}$ is slope stable. A semistable sheaf that is not stable is said to be strictly semi-stable. An equivalent formulation of semi-stability is that $\mu(\mathcal{I}) \leq \mu(\mathcal{J})$ for all quotients $\mathcal{I} \rightarrow \mathcal{J}$ with $\mathcal{J}$ pure of dimension $d(\mathcal{J})=d(\mathcal{I})$, and similarly with stability.

We have just given the general definition of semi-stability, but observe that, on a ribbon, the condition is independent of $\mathcal{L}$. Indeed, the slope $\mu(\mathcal{I})$ depends on $\mathcal{L}$, but replacing $\mathcal{L}$ with a different ample line bundle $\mathcal{M}$ 
modifies the slope by a factor of $\operatorname{deg}(\mathcal{L}) / \operatorname{deg}(\mathcal{M})$, so the slope semi-stability condition is unchanged.

Given a semi-stable sheaf $\mathcal{I}$, there exists a filtration $0=\mathcal{I}_{0} \subset \cdots \subset \mathcal{I}_{n}=\mathcal{I}$ with the property that the successive quotients $\mathcal{I}_{k} / \mathcal{I}_{k-1}$ are slope stable of dimension $d(\mathcal{I})$ and the slopes $\mu\left(\mathcal{I}_{k} / \mathcal{I}_{k-1}\right)$ are all equal. If $\mathcal{I}$ is stable, this filtration is trivial. If $\mathcal{I}$ is strictly semi-stable, this filtration may not be unique, but the associated direct sum

$$
\operatorname{Gr}(\mathcal{I}):=\bigoplus_{k} \mathcal{I}_{k} / \mathcal{I}_{k-1}
$$

is unique up to isomorphism. Two strictly semi-stable coherent sheaves $\mathcal{I}$ and $\mathcal{I}^{\prime}$ are said to be $G r$-equivalent if there is an isomorphism $\operatorname{Gr}(\mathcal{I}) \cong$ $\operatorname{Gr}\left(\mathcal{I}^{\prime}\right)$. Given a semi-stable sheaf $\mathcal{I}$, observe that $\operatorname{Gr}(\mathcal{I})$ is also a semi-stable sheaf whose associated direct sum is $\operatorname{Gr}(\mathcal{I})$.

We now specialize to the case of semi-stable sheaves on $X$ with Hilbert polynomial

$$
P_{d}(t):=\operatorname{deg}(\mathcal{L}) t+d+1-g .
$$

As said before, this is the Hilbert polynomial of a degree $d$ generalized line bundle. We will show that the stability condition on a generalized line bundle is controlled by a distinguished quotient. Following [EG95, p. 759], we make the following definition.

Definition 3.1. The sheaf $\overline{\mathcal{I}}$ associated to a generalized line bundle $\mathcal{I}$ is defined to be the maximal torsion-free quotient of $\mathcal{I} \otimes \mathcal{O}_{X_{\text {red }}}$, i.e., $\overline{\mathcal{I}}=$ $\left(\left.\mathcal{I}\right|_{X_{\text {red }}} /\right.$ torsion $)$.

By Fact 2.8 , the sheaf $\overline{\mathcal{I}}$ is a line bundle on $X_{\text {red }}$ of degree $(\operatorname{deg}(\mathcal{I})-$ $b(\mathcal{I})) / 2$. By the projection formula, the Hilbert polynomial of $i_{*}(\overline{\mathcal{I}})$ with respect to $\mathcal{L}$ is equal to the Hilbert polynomial of $\overline{\mathcal{I}}$ with respect to $i^{*}(\mathcal{L})$. This common polynomial is

$$
\frac{\operatorname{deg}(\mathcal{L})}{2} t+(\operatorname{deg}(\mathcal{I})-b(\mathcal{I})) / 2+1-\bar{g}
$$

Using $\overline{\mathcal{I}}$ as a test quotient sheaf, we conclude that if $\mathcal{I}$ is semi-stable, then its index must satisfy

$$
b(\mathcal{I}) \leq 1+g-2 \bar{g} .
$$

In fact, this inequality characterizes slope semi-stability.

Lemma 3.2. Let $(X, \mathcal{L})$ be a polarized ribbon and $\mathcal{I}$ a generalized line bundle of degree $d$. Then $\mathcal{I}$ is slope semi-stable with respect to $\mathcal{L}$ if and only if Inequality (3.3) holds. Similarly, $\mathcal{I}$ is slope stable if and only if Inequality (3.3) is strict.

Proof. Inequality (3.3) is equivalent to the slope inequality $\mu(\mathcal{I}) \leq \mu(\overline{\mathcal{I}})$, so one implication is clear. For the converse, we assume $\mu(\mathcal{I}) \leq \mu(\overline{\mathcal{I}})$ and then prove $\mu(\mathcal{I}) \leq \mu(\mathcal{J})$ for all pure quotients $q: \mathcal{I} \rightarrow \mathcal{J}$ with $d(\mathcal{J})=1$. 
There are two separate cases to consider: the case where the leading term of $P(\mathcal{J}, t)$ is $\operatorname{deg}(\mathcal{L}) \cdot t$ and the case where it is $\operatorname{deg}(\mathcal{L}) / 2 \cdot t$.

First, suppose the leading term of $P(\mathcal{J}, t)$ is $\operatorname{deg}(\mathcal{L}) \cdot t$. We claim that $q$ is in fact an isomorphism, and thus there is no slope inequality to check. We begin by showing that $\mathcal{J}$ is a generalized line bundle. The condition on the Hilbert polynomial is equivalent to the condition that the generic stalk $\mathcal{J}_{\eta}$ has length 2 , so by Proposition 2.6 it is enough to show that $\mathcal{J}$ is not the direct image of a rank 2 vector bundle on $X_{\text {red }}$. This, however, is clear: $\mathcal{J}_{\eta}$ is generated by a single element (the image of a local generator of $\mathcal{I}$ ), but the direct image of a rank 2 vector bundle does not have this property. Having shown that $\mathcal{J}$ is a generalized line bundle, the result follows easily. Consider the kernel of $q$. Because both $\mathcal{I}$ and $\mathcal{J}$ are generalized line bundles, $q$ is generically an isomorphism, hence $\operatorname{ker}(q)$ is generically zero. $\operatorname{But} \operatorname{ker}(q)$ is a subsheaf of the pure sheaf $\mathcal{I}$, so this forces $\operatorname{ker}(q)=0$. We can conclude that $q$ is an isomorphism.

Next we consider the case where the leading term of $P(\mathcal{J}, t)$ is $\operatorname{deg}(\mathcal{L}) / 2 \cdot t$. To begin, we claim that the kernel $\mathcal{K}$ of $\mathcal{O}_{X} \rightarrow \underline{\operatorname{End}}(\mathcal{J})$ equals $\mathcal{N}$. Consider the generic stalk $\mathcal{J}_{\eta}$. The assumption on the Hilbert polynomial implies that the length of $\mathcal{J}_{\eta}$, and hence of every non-zero cyclic submodule, is 1 . In particular, if $s_{0} \in \mathcal{J}_{\eta}$ is non-zero, then $\mathcal{O}_{X, \eta} \cdot s_{0}=\mathcal{J}_{\eta} / \operatorname{ann}\left(s_{0}\right)$ has length 1. Since $\mathcal{I}$ has length 2 , we may conclude that $\mathcal{K}_{\eta}=\operatorname{ann}\left(s_{0}\right)$ is non-zero. By Lemma 2.4, we can conclude that $\mathcal{K}=\mathcal{N}$.

Because $\mathcal{K}=\mathcal{N}$, we may factor $q$ as $\mathcal{I} \rightarrow i_{*}(\overline{\mathcal{I}}) \stackrel{\bar{q}}{\rightarrow} \mathcal{J}$. Furthermore, consider $\mathcal{J}$ as a module over $\mathcal{O}_{X} / \mathcal{N}=\mathcal{O}_{X_{\text {red }}}$, which is also a line bundle on $X_{\text {red }}$ because it is locally free (as it is pure) of generic rank 1 . Thus, $\bar{q}$ is a surjection between line bundles on $X_{\text {red }}$, so it must be an isomorphism. In particular, the inequality $\mu(\mathcal{I}) \leq \mu(\mathcal{J})$ is exactly the hypothesis of the lemma. This completes the proof.

The lemma also shows that the strictly semi-stable generalized line bundles are exactly the generalized line bundles of index $b(\mathcal{I})=1+g-2 \bar{g}$. It is natural to ask what their associated graded modules are.

Lemma 3.3. Let $(X, \mathcal{L})$ be a polarized ribbon and $\mathcal{I}$ a generalized line bundle of index $b(\mathcal{I})=1+g-2 \bar{g}$. Set $\mathrm{F}_{1}(\mathcal{I})$ to be the kernel of $\mathcal{I} \rightarrow \overline{\mathcal{I}}$. Then $0 \subset \mathrm{F}_{1}(\mathcal{I}) \subset \mathcal{I}$ is a filtration whose successive quotients are stable with the same Hilbert polynomial, and

$$
\operatorname{Gr}(\mathcal{I})=\mathrm{F}_{1}(\mathcal{I}) \oplus \overline{\mathcal{I}}
$$

Proof. Both $\mathrm{F}_{1}(\mathcal{I})$ and $\overline{\mathcal{I}}$ are pure of dimension 1 (in fact, direct images of the line bundles on $\left.X_{\text {red }}\right)$, so it is enough to show $\mu(\overline{\mathcal{I}})=\mu\left(\mathrm{F}_{1}(\mathcal{I})\right)$. This follows from the work we have already shown. One computes

$$
\begin{gathered}
\mu(\overline{\mathcal{I}})=(\operatorname{deg}(\mathcal{I})-b(\mathcal{I})+2-2 \bar{g}) / \operatorname{deg}(\mathcal{L}), \\
\mu\left(\mathrm{F}_{1}(\mathcal{I})\right)=(\operatorname{deg}(\mathcal{I})+b(\mathcal{I})+2 \bar{g}-2 g) / \operatorname{deg}(\mathcal{L}) .
\end{gathered}
$$


It is easy to check that these two numbers are equal precisely when $b(\mathcal{I})=$ $1+g-2 \bar{g}$.

The submodule $\mathrm{F}_{1}(\mathcal{I})$ can be described more explicitly. Say the blow-up $f: X^{\prime} \rightarrow X$ associated to $\mathcal{I}$ is given by blowing up the divisor $D \subset X_{\text {red }}$. Then $\mathrm{F}_{1}(\mathcal{I})=\mathcal{N} \otimes \overline{\mathcal{I}}(D)$. (This is [EG95, p. 759, bottom of page]).

$\mathrm{F}_{1}(\mathcal{I}) \oplus \overline{\mathcal{I}}$ is, of course, the direct image of a split rank 2 vector bundle on $X_{\text {red }}$. For the sake of completeness, we should also discuss the slope stability condition on the direct image $i_{*}(\mathcal{E})$ of a vector bundle, but there is little to say. Slope stability of $i_{*}(\mathcal{E})$ is equivalent to slope stability of $\mathcal{E}$. This will be used in a later section, so let us record it as a fact.

Fact 3.4. Let $X$ be a ribbon. Then the direct image $i_{*} \mathcal{E}$ of a rank 2 vector bundle $\mathcal{E}$ on $X_{\text {red }}$ is semi-stable if and only if for every subbundle $\mathcal{F} \subset \mathcal{E}$, we have

$$
\operatorname{deg}(\mathcal{F}) / \operatorname{rank}(\mathcal{F}) \leq \operatorname{deg}(\mathcal{E}) / \operatorname{rank}(\mathcal{E}) .
$$

In particular, if $X$ is a rational ribbon (i.e. $X_{\mathrm{red}} \cong \mathbb{P}^{1}$ ), then there are no stable sheaves of this form and the only semi-stable sheaves are $i_{*}(\mathcal{O}(e) \oplus$ $\mathcal{O}(e))$.

We summarize the results of this section with the following theorem.

Theorem 3.5. Let $X$ be a ribbon of genus $g$ with a polarization $\mathcal{L}$. Let $\mathcal{I}$ be a coherent sheaf on $X$ whose generic stalk has length 2 . Then $\mathcal{I}$ is pure and slope (semi-) stable with respect to $\mathcal{L}$ if and only if it is one of the following sheaves:

(1) a generalized line bundle with index $b$ satisfying $(b \leq 1+g-2 \bar{g})$ $b<1+g-2 \bar{g}$

(2) the direct image $i_{*}(\mathcal{E})$ of a (semi-) stable rank 2 vector bundle $\mathcal{E}$ on $X_{\text {red }}$.

In particular, slope stability of $\mathcal{I}$ is independent of $\mathcal{L}$.

The special case of rational ribbons is particularly nice.

Corollary 3.6. Let $(X, \mathcal{L})$ be a polarized rational ribbon. If $\mathcal{I}$ is a slope semi-stable sheaf with Hilbert polynomial $P_{d}(t)$, then it is one of the following sheaves:

(1) a degree $d$ generalized line bundle whose index satisfies $b(\mathcal{I}) \leq 1+g$;

(2) the direct image $i_{*}(\mathcal{E})$, where $\mathcal{E}=\mathcal{O}((d-1-g) / 2) \oplus \mathcal{O}((d-1-g) / 2)$.

The second case can only occur when $d-1-g$ is even.

If $\mathcal{I}$ is strictly semi-stable, then it is one of the following sheaves:

(1) a degree d generalized line bundle whose index satisfies $b(\mathcal{I})=g+1$;

(2) the direct image $i_{*}(\mathcal{E})$, where $\mathcal{E}=\mathcal{O}((d-1-g) / 2) \oplus \mathcal{O}((d-1-g) / 2)$.

In particular, any two strictly semi-stable sheaves are Gr-equivalent, and if $d-1-g$ is odd, then slope semi-stability is equivalent to slope stability. 


\section{Moduli of Sheaves on a RibBon}

Semi-stable generalized line bundles were described in Section 3. The significance of these sheaves is that their Gr-equivalence classes are are coarsely parameterized by a moduli space. Simpson [Sim94] has constructed the coarse moduli space of semi-stable sheaves up to Gr-equivalence on an arbitrary polarized projective $k$-scheme, in particular on a ribbon. In his paper, Simpson works over the complex numbers, but his work has since been generalized to positive characteristic ([Mar96, Thm. 0.6] or [Lan04, Thm. 0.2]). Here we describe the geometry of the Simpson moduli space. As before, $X$ will be a ribbon of genus $g$ with nilradical $\mathcal{N}$. We write $\bar{g}$ for the genus of $X_{\text {red }}$.

On a ribbon, we have seen that the slope stability condition is independent of the choice of polarization, but to fix ideas, let us temporarily work with a polarized ribbon $(X, \mathcal{L})$. Given a polynomial $P(t)$, the Simpson moduli functor $\mathrm{M}^{\sharp}\left(\mathcal{O}_{X}, P\right): k$-Sch $\rightarrow$ Sets is defined by letting the set of $T$-valued points equal the set of isomorphism classes of $\mathcal{O}_{T}$-flat, finitely presented $\mathcal{O}_{X_{T}}$-modules that are fiber-wise slope semi-stable with Hilbert polynomial $P(t)$. Inside of the Simpson moduli functor, we can consider the subfunctor $\mathrm{M}_{\mathrm{S}}^{\sharp}\left(\mathcal{O}_{X}, P\right)$ that parameterizes slope stable sheaves.

The basic existence theorem [Sim94, Thm. 1.21] states that there exists a pair $\left(\mathrm{M}\left(\mathcal{O}_{X}, P\right), p\right)$ consisting of a projective scheme $\mathrm{M}\left(\mathcal{O}_{X}, P\right)$ and a natural transformation $p: \mathrm{M}^{\sharp}\left(\mathcal{O}_{X}, P\right) \rightarrow \mathrm{M}\left(\mathcal{O}_{X}, P\right)$ that universally corepresents $\mathrm{M}^{\sharp}\left(\mathcal{O}_{X}, P\right)$. In other words, $p$ is universal with respect to natural transformations from $\mathrm{M}^{\sharp}\left(\mathcal{O}_{X}, P\right)$ to a $k$-scheme and this property persists under base change by an arbitrary morphism $T \rightarrow \mathrm{M}\left(\mathcal{O}_{X}, P\right)$. Furthermore, $p$ induces a bijection between the $k$-valued points of $\mathrm{M}\left(\mathcal{O}_{X}, P\right)$ and the set of Gr-equivalence classes of semi-stable sheaves on $X$ with Hilbert polynomial $P(t)$. We call $\mathrm{M}\left(\mathcal{O}_{X}, P\right)$ the Simpson moduli space.

Restricting to stable sheaves, there is an open subscheme $\mathrm{M}_{\mathrm{S}}\left(\mathcal{O}_{X}, P\right)$ of $\mathrm{M}\left(\mathcal{O}_{X}, P\right)$ whose pre-image under $p$ is $\mathrm{M}_{\mathrm{s}}^{\sharp}\left(\mathcal{O}_{X}, P\right)$. The restriction $\mathrm{M}_{\mathrm{s}}^{\sharp}\left(\mathcal{O}_{X}, P\right) \rightarrow$ $\mathrm{M}_{\mathrm{s}}\left(\mathcal{O}_{X}, P\right)$ realizes $\mathrm{M}_{\mathrm{s}}\left(\mathcal{O}_{X}, P\right)$ as the scheme that represents the étale sheaf associated to $\mathrm{M}_{\mathrm{S}}\left(\mathcal{O}_{X}, P\right)$. (This is a restatement of [Sim94, Thm. 1.21(4)].) The scheme $\mathrm{M}_{\mathrm{s}}\left(\mathcal{O}_{X}, P\right)$ is called the stable Simpson moduli space.

If we specialize to the case where $P(t)$ equals $P_{d}(t):=\operatorname{deg}(\mathcal{L}) t+d+1-g$, the Simpson moduli space can be described using the results from Section 3: it is the coarse moduli space of Gr-equivalence classes of length 2, semi-stable sheaves of degree $d$. The stable locus is the fine moduli space parameterizing those sheaves that are stable. In [EG95], Eisenbud and Green asked if it is possible to compactify the Jacobian of a rational ribbon by a moduli space parameterizing generalized line bundles of index at most $g$. The Simpson moduli space is a natural candidate for such a compactification.

Corollary 4.1 (Reformulation of Corollary 3.6). Let $X$ be a rational ribbon. Then the stable Simpson moduli space $\mathrm{M}_{\mathrm{S}}\left(\mathcal{O}_{X}, P_{0}\right)$ parameterizes generalized line bundles of degree 0 and index at most $g$. If $g$ is even, then $\mathrm{M}_{\mathrm{s}}\left(\mathcal{O}_{X}, P_{0}\right)$ 
is projective. If $g$ is odd, the complement of the stable locus in $\mathrm{M}\left(\mathcal{O}_{X}, P_{0}\right)$ consists of a single point that represents the Gr-equivalence class of the sheaf

$$
\left.i_{*}(\mathcal{O}((-1-g) / 2) \oplus(-1-g) / 2)\right) .
$$

This equivalence class consists of the above sheaf and every generalized line bundle of degree 0 and index $g+1$.

We now turn our attention to describing the global and local geometry of the Simpson moduli space.

4.1. Global Structure. Having defined $\mathrm{M}\left(\mathcal{O}_{X}, P_{d}\right)$, we study the global geometry of this space. First, observe that it is impossible for a rank 2 vector bundle on $X_{\text {red }}$ to specialize to a generalized line bundle on $X$.

Lemma 4.2. Suppose $T$ is a $k$-scheme and $\mathcal{I}$ a sheaf that represents a $T$ valued point of $\mathrm{M}^{\sharp}\left(\mathcal{O}_{X}, P\right)$. Define $T_{0} \subset T$ to be the locus of points $t \in T$ with the property that the restriction of $\mathcal{I}$ to the fiber $X_{t}:=X_{T} \times_{T} \operatorname{Spec}(k(t))$ is a generalized line bundle. Then $T_{0} \subset T$ is open.

Proof. By [Sim94], the moduli space of semi-stable vector bundles on $X_{\text {red }}$ is projective, hence is universally closed. It follows that $T \backslash T_{0}$ is closed.

Next, we compute the dimension of various loci of generalized line bundles in $\mathrm{M}\left(\mathcal{O}_{X}, P\right)$.

Definition 4.3. Let $X$ be a ribbon of genus $g$ and $\underline{b}:=\left(b_{1}, \ldots, b_{k}\right)$ a (possibly empty) sequence of positive integers satisfying $b_{1}+\cdots+b_{k} \leq g-2 \bar{g}$. Define $Z_{\underline{b}} \subset \mathrm{M}_{\mathrm{s}}\left(\mathcal{O}_{X}, P_{d}\right)$ to be the subset of stable generalized line bundles of degree $d$ whose local index sequence equals $\underline{b}$.

Lemma 4.4. If $d-b_{1}-\cdots-b_{k}$ is odd, then $Z_{\underline{b}}$ is empty. Otherwise, it is a locally closed, irreducible subset of dimension

$$
\operatorname{dim}\left(Z_{\underline{b}}\right)=g-\left(b_{1}-1\right)-\cdots-\left(b_{k}-1\right) .
$$

Proof. Set $b:=b_{1}+\cdots+b_{k}$, the index of a generalized line bundle with local index sequence $\underline{b}$. When $d-b$ is odd, the claim is follows from Fact 2.8 . Thus, assume that $d-b$ is even. We prove the lemma by parameterizing $Z_{\underline{b}}$ by an irreducible variety of the appropriate dimension.

Let $U \subset \operatorname{Hilb}^{b}\left(X_{\text {red }}\right)$ denote the subset parameterizing closed subschemes $\Sigma$ supported at $k$ distinct points with multiplicities $b_{1}, \ldots, b_{k}$. The ideal $\mathcal{I}_{\Sigma}$ in $\mathcal{O}_{X}$ of such a subscheme is a generalized line bundle of degree $-b$ with local index sequence $\underline{b}$ by Fact 2.15 . Furthermore, $U$ is locally closed and irreducible of dimension $k$, because it is isomorphic to an open subset of the $k$-th symmetric power of $X_{\text {red }}$. The hypothesis on $b$ implies that a generalized line bundle of index $b$ is stable, so we can define a morphism $U \times \operatorname{Pic}^{d+b}(X) \rightarrow \mathrm{M}\left(\mathcal{O}_{X}, P_{d}\right)$ by the rule $(\Sigma, \mathcal{L}) \mapsto \mathcal{I}_{\Sigma} \otimes \mathcal{L}$.

Observe that $U$ has been chosen so that if $p_{1}, \ldots, p_{k}$ is a collection of $k$ distinct points, then there is a unique closed subscheme $\Sigma$ corresponding to a point of $U$ with the property that $b_{p_{i}}\left(\mathcal{I}_{\Sigma}\right)=b_{i}$. By Lemma 2.9, we 
may deduce that the image of $U \times \operatorname{Pic}^{d+b}(X) \rightarrow \mathrm{M}\left(\mathcal{O}_{X}, P_{d}\right)$ is $Z_{\underline{b}}$. Note that the fiber over a point is an irreducible variety of dimension $b$, given by $\operatorname{ker}\left(f^{*}: \operatorname{Pic}(X) \rightarrow \operatorname{Pic}\left(X^{\prime}\right)\right)$ where $f: X^{\prime} \rightarrow X$ is the associated blow-up. We can immediately conclude that $Z_{b}$ is irreducible and constructible.

Furthermore, using the formula $b \overline{(\mathcal{I}})=\operatorname{len}\left(\underline{\operatorname{End}}(\mathcal{I}) / \mathcal{O}_{X}\right)$ for index, it is easy to see that the index of a generalized line bundle can only increase under specialization. Because $Z_{\underline{b}}$ is constructible, it follows that this subset is locally closed. To complete the proof, we compute the dimension of $Z_{\underline{b}}$ :

$$
\begin{aligned}
\operatorname{dim}\left(Z_{\underline{b}}\right) & =\operatorname{dim}\left(U \times \operatorname{Pic}_{X}^{d+b}\right)-\operatorname{dim}(\text { Fiber }) \\
& =(g+k)-b .
\end{aligned}
$$

Lemma 4.5. Let $X$ be a ribbon and $\mathcal{I}$ a generalized line bundle. Say that $p_{0} \in X$ is a point such that $b_{p_{0}}(\mathcal{I})=b_{0}+2$ for some $b_{0} \geq 0$. Then there is a $\operatorname{Spec}(k[\alpha])$-flat, finitely presented $\mathcal{O}_{X \times \operatorname{Spec}(k[\alpha])}$-module $\mathcal{J}$ whose special fiber is isomorphic to $\mathcal{I}$ and whose generic fiber $\mathcal{I}_{\alpha}$ for general $\alpha$ is a generalized line bundle with local index

$$
b_{p}\left(\mathcal{I}_{\alpha}\right)= \begin{cases}b_{p}(\mathcal{I}) & \text { if } p \neq p_{0} \\ b_{0} & \text { if } p=p_{0}\end{cases}
$$

Proof. Given $\mathcal{I}$, we first exhibit another generalized line bundle $\mathcal{I}^{\prime}$ with the same local index as $\mathcal{I}$ at every point $p \in X$ that admits a suitable deformation. Then we use Lemma 2.9 to deform $\mathcal{I}$.

Suppose that $p_{0}, p_{1}, \ldots, p_{k}$ are the points $p$ with $b_{p}(\mathcal{I}) \neq 0$. Define $Z$ to be the (unique) closed subscheme of $X_{\text {red }}$ that is supported at $p_{0}, p_{1}, \ldots, p_{k}$ and has length $b_{p_{i}}(\mathcal{I})$ at $p_{i}$. If we consider $Z$ as a closed subscheme of $X$, then the ideal sheaf $\mathcal{I}^{\prime}:=\mathcal{I}_{Z}$ is a generalized line bundle with the property that $b_{p}(\mathcal{I})=b_{p}\left(\mathcal{I}^{\prime}\right)$ for all $p \in X$. We deform $\mathcal{I}^{\prime}$ by deforming $Z$ as a closed subscheme.

Fix an open affine neighborhood $U$ of $p_{0}$ with the property that $p_{i} \notin U$ for $i \neq 0$ and $\left.Z\right|_{U}$ (considered as a subscheme of $X$ ) is defined by $\left(\epsilon, s^{b_{0}+2}\right)$ for regular functions $\epsilon, s$ with $\epsilon$ generating the nilradical. We can explicitly deform $\left(\epsilon, s^{b_{0}+2}\right)$ as follows. By e.g. properness of the Hilbert scheme, we can extend the ideal $\left(\epsilon, s^{b_{0}}\right) \cap\left(\epsilon-\alpha^{b_{0}+1}(s-\alpha),(s-\alpha)^{2}\right)$ to a proper flat family $\mathcal{J}^{\prime}$ over Spec $k[\alpha]$. We claim that this extension is the desired deformation, i.e. that the fiber over $0 \in \operatorname{Spec} k[\alpha]$ is $\mathcal{I}^{\prime}$.

To verify the claim, observe that the intersection $\left(\epsilon, s^{b_{0}}\right) \cap\left(\epsilon-\alpha^{b_{0}+1}(s-\right.$ $\left.\alpha),(s-\alpha)^{2}\right)$ contains $s^{b_{0}}(s-\alpha)^{2}$ and

$\epsilon-\alpha s^{b_{0}+1}+\alpha^{2} s^{b_{0}}=\epsilon-\alpha^{b_{0}+1}(s-\alpha)-(s-\alpha)^{2} \alpha\left(\alpha^{b_{0}-1}+\alpha^{b_{0}-2} s+\cdots+s^{b_{0}-1}\right)$.

These two elements must lie in the general fiber $\mathcal{I}_{\alpha}^{\prime}$ of $\mathcal{J}^{\prime}$, so the fiber over 0 must contain $\left(s^{b_{0}+2}, \epsilon\right)$, and hence equal $\left(s^{b_{0}+2}, \epsilon\right)$ by degree considerations. For general $\alpha$, the ideal $\left(\epsilon-\alpha^{b_{0}+1}(s-\alpha),(s-\alpha)^{2}\right)$ defines a double point at $s=\alpha$, which is Cartier on $X$, hence it has index 0 . This explains where the 
extra 2 goes from the exponent of $s$ in $\mathcal{I}^{\prime}$. The ideal $\left(\epsilon, s^{b_{0}}\right)$ has local index $b_{0}$ at $p_{0}$. Therefore, $\mathcal{I}_{\alpha}^{\prime}$ is the desired deformation of $\mathcal{I}^{\prime}$.

This proves the lemma when $\mathcal{I}=\mathcal{I}^{\prime}$. To deduce the general case, by Lemma 2.9 there exists a line bundle $\mathcal{L}$ such that $\mathcal{I}=\mathcal{I}^{\prime} \otimes \mathcal{L}$. If we define $\mathcal{J}$ to be the tensor product of $\mathcal{J}^{\prime}$ with the constant family with fiber $\mathcal{L}$, then $\mathcal{J}$ satisfies the conditions of the lemma. This completes the proof.

Theorem 4.6. Let $X$ be a ribbon and $i$ an integer satisfying

$$
1 \leq i \leq \begin{cases}(g+2) / 2-\bar{g} & \text { if } d \text { is even } \\ (g+1) / 2-\bar{g} & \text { if } d \text { is odd }\end{cases}
$$

Define $\bar{Z}_{i} \subset \mathrm{M}\left(\mathcal{O}_{X}, P_{d}\right)$ to be the Zariski closure of $Z_{\underline{b}}$, where $\underline{b}=(1, \ldots, 1)$ is the sequence of 1 's with length equal to $2 i-2$ if $d$ is even and $2 i-1$ if $d$ is odd.

Then $\bar{Z}_{i}$ is a g-dimensional irreducible component of $\mathrm{M}\left(\mathcal{O}_{X}, P_{d}\right)$. Furthermore, if $\bar{Z} \subset \mathrm{M}\left(\mathcal{O}_{X}, P_{d}\right)$ is any irreducible component that contains a stable generalized line bundle, then $\bar{Z}=\bar{Z}_{i}$ for some $i$.

Proof. The theorem follows from Lemmas 4.2, 4.4, and 4.5. Observe that a repeated application of Lemma 4.5 shows that $\cup \bar{Z}_{i}$ contains the locus of generalized line bundles. Furthermore, the subsets $\bar{Z}_{i}$ are all irreducible and of dimension $g$ by Lemma 4.4. We now use these facts to prove the theorem.

First, let us prove that every irreducible component that contains a stable generalized line bundle is of the form $\bar{Z}_{i}$ for some $i$. Say $\bar{Z}$ is an irreducible component that contains a stable generalized line bundle. The subset of $\bar{Z}$ consisting of stable generalized line bundles is non-empty and open (Lemma 4.2), hence dense. We may conclude that $\bar{Z}$ is contained in the union $\cup \bar{Z}_{j}$, but each of the $\bar{Z}_{j}$ 's is irreducible, so this is only possible if $\bar{Z}=\bar{Z}_{i}$ for some $i$.

This shows that some of the $\bar{Z}_{i}$ 's are irreducible components of $\mathrm{M}\left(\mathcal{O}_{X}, P_{d}\right)$. To complete the proof, we must show that every $\bar{Z}_{i}$ is a component, so let $\bar{Z}_{i}$ be given. Certainly this subset is contained in some component, which we have shown must be of the form $\bar{Z}_{j}$ for some $j$. In other words, we have $\bar{Z}_{i} \subset \bar{Z}_{j}$. As both are irreducible of dimension $g$, this is only possible if $\bar{Z}_{i}=\bar{Z}_{j}$, showing that $\bar{Z}_{i}$ is a component. This completes the proof.

Theorem 4.6 describes all the irreducible components of $\mathrm{M}\left(\mathcal{O}_{X}, P_{d}\right)$ that contain a stable generalized line bundle. What about the locus parameterizing sheaves of the form $i_{*} \mathcal{E}$ for $\mathcal{E}$ a semi-stable rank 2 vector bundle on $X_{\text {red }}$ ? We prove the following result.

Theorem 4.7. Let $X$ be a ribbon of genus $g$.

(1) If $g \leq 2 \bar{g}-1$, then no component of $\mathrm{M}\left(\mathcal{O}_{X}, P_{d}\right)$ contains a stable generalized line bundle. Furthermore, the moduli space is empty when 
$\bar{g}=0$ and $d-g$ is even. Otherwise $\mathrm{M}\left(\mathcal{O}_{X}, P_{d}\right)$ is irreducible and

$$
\operatorname{dim} \operatorname{M}\left(\mathcal{O}_{X}, P_{d}\right)= \begin{cases}0 & \text { if } \bar{g}=0 ; \\ 1 & \text { if } \bar{g}=1, d-g \text { is even } ; \\ 2 & \text { if } \bar{g}=1, d-g \text { is odd } \\ 4 \bar{g}-3 & \text { if } \bar{g} \geq 2 .\end{cases}
$$

(2) If $g>2 \bar{g}-1$, then $\mathrm{M}\left(\mathcal{O}_{X}, P_{d}\right)$ has at most one irreducible component that does not contain a stable generalized line bundle. When this component exists, it has dimension $4 \bar{g}-3$. This component does not exist when $\bar{g} \leq 1$, but does exist when $\bar{g} \geq 2$ and $4 \bar{g}-3 \geq g$.

Theorem 4.7 follows from known results about moduli of vector bundles on non-singular curves, so before giving the proof, we recall the relevant facts from [LP95].

Computing Euler characteristics, we see that the direct image of a rank 2 semi-stable vector bundle $\mathcal{E}$ on $X_{\text {red }}$ corresponds to a point of $\mathrm{M}\left(\mathcal{O}_{X}, P_{d}\right)$ precisely when $\operatorname{deg}(\mathcal{E})=d+2 \bar{g}-g-1$. Thus, if we set

$$
e:=d+2 \bar{g}-g-1
$$

and $\mathrm{M}(2, e)$ equal to the coarse moduli space of Gr-equivalence classes of semi-stable rank 2 vector bundles on $X_{\text {red }}$ of degree $e$ (which exists by, say, Simpson's work), then the rule $\mathcal{E} \mapsto i_{*} \mathcal{E}$ defines a closed embedding $\mathrm{M}(2, e) \rightarrow \mathrm{M}\left(\mathcal{O}_{X}, P_{d}\right)$ with image equal to the subset parameterizing sheaves of the form $i_{*} \mathcal{E}$. The geometry of $\mathrm{M}(2, e)$ depends on the genus of $X_{\text {red }}$. There are three cases to consider: $\bar{g}=0, \bar{g}=1$ and $\bar{g} \geq 2$.

When $\bar{g}=0$, every rank 2 vector bundle splits as a direct sum of line bundles, so there are no stable vector bundles and the strictly semi-stable vector bundles are of the form $\mathcal{O}_{\mathbb{P}^{1}}(e / 2) \oplus \mathcal{O}_{\mathbb{P}^{1}}(e / 2)$ for $e$ even. Thus, $\mathrm{M}(2, e)$ is empty when $e$ is odd (i.e. $d-g$ is even) and equal to a point when $e$ is even (i.e. $d-g$ is odd).

The next case to consider is $\bar{g}=1$. Again, the geometry of $\mathrm{M}(2, e)$ depends on the parity of $e$. For $e$ odd (i.e. $d-g$ even), every semi-stable rank 2 vector bundle of degree $e$ is stable and the determinant map defines an isomorphism $\mathrm{M}(2, e) \rightarrow \operatorname{Pic}^{e}\left(X_{\text {red }}\right)$ ([LP95, Thm. 8.29]). In particular, $\mathrm{M}(2, e)$ is irreducible of dimension 1 . By contrast, there are no stable vector bundles of odd degree on $X_{\text {red }}$ ([LP95, p. 160-161]), and M(2,e) is irreducible of dimension 2 ([LP95, Thm. 8.29]).

The final case is $\bar{g} \geq 2$. In this case, there always exists a stable rank 2 vector bundle of degree $e$ ([LP95, Thm. 8.28]), and $\mathrm{M}(2, e)$ is irreducible of dimension $4 \bar{g}-3$ ([LP95, Thm. 8.14, 8.26]). We now use these facts to prove the theorem.

Proof of Theorem 4.7. The case where $g \leq 2 \bar{g}-1$ follows from Theorem 3.5. That theorem asserts that there are no stable generalized line bundles, so the natural map $\mathrm{M}(2, e) \rightarrow \mathrm{M}\left(\mathcal{O}_{X}, P_{d}\right)$ is a set-theoretic bijection, and the claim 
follows from the results about $\mathrm{M}(2, e)$ just reviewed. For the remainder of the proof, we assume $g>2 \bar{g}-1$.

The case $\bar{g}=0$ can be treated without difficulty. By the classification of vector bundles on $\mathbb{P}^{1}$, it is enough to show that if $d-g$ is odd, then the point of $\mathrm{M}\left(\mathcal{O}_{X}, P_{d}\right)$ corresponding to the direct image of

$$
\mathcal{O}(e / 2) \oplus \mathcal{O}(e / 2)
$$

is contained in the closure of the locus of generalized line bundles. The vector bundle in question is Gr-equivalent to a generalized line bundle of index $g+1$, and every such generalized line bundle is the specialization of a stable generalized line bundle by Lemma 4.5, combined with Corollary 3.6.

We now turn our attention to the case where $\bar{g}=1$. When $d-g$ is odd, the claim follows from essentially the same argument as in the $\bar{g}=0$ : there are no stable vector bundles of degree $e$ on $X_{\text {red }}$ and every strictly semistable rank 2 vector bundle is Gr-equivalent to a generalized line bundle of index $g-1$. However, when $d-g$ is even, a different argument is needed.

Consider first the special case where we not only assume $\bar{g}=1$ and $d-g$ is even, but also $g=2$. Then there are exactly two types of semi-stable sheaves with degree $d$ : line bundles on $X$ and sheaves of the form $i_{*} \mathcal{E}$ for $\mathcal{E}$ a rank 2 stable vector bundle on $X_{\text {red }}$. The moduli space $\operatorname{Pic}^{d}(X)$ is not projective, hence its image in $\mathrm{M}\left(\mathcal{O}_{X}, P_{d}\right)$ is not closed (because $\mathrm{M}\left(\mathcal{O}_{X}, P_{d}\right)$ is projective). Therefore, its closure in $\mathrm{M}\left(\mathcal{O}_{X}, P_{d}\right)$ must contain some stable vector bundle. But the natural action of $\mathrm{Pic}^{0}(X)$ on the set of stable rank 2 vector bundles of degree $e$ is transitive, so if the closure of $\operatorname{Pic}^{d}(X)$ contains one such vector bundle, then it contains all such vector bundles. This completes the proof when $g=2$.

The case where $g>2$ can be deduced from this. Suppose now that we relax the condition $g=2$ to $g \geq 2$. Blowing-up a length $g-2$ closed subscheme contained in $X_{\text {red }}$, we obtain a finite morphism $f: X^{\prime} \rightarrow X$ with $g\left(X^{\prime}\right)=2$. Now suppose that we are given a sheaf of the form $i_{*} \mathcal{E}$ for $\mathcal{E}$ a stable rank 2 vector bundle on $X_{\text {red }}$. We need to show that $i_{*} \mathcal{E}$ is the specialization of a stable generalized line bundle. By what we have shown, there exists a family of sheaves $\mathcal{I}^{\prime}$ on $X^{\prime}$ whose generic fiber is a stable line bundle and whose special fiber is isomorphic to $i_{*} \mathcal{E}$. The direct image of this family under $f$ realizes $i_{*} \mathcal{E}$ as the specialization of stable generalized line bundles. This completes the proof in the case that $\bar{g}=1$.

The remaining case is $\bar{g} \geq 2$ and $4 \bar{g}-3 \geq g$. Under these conditions, we wish to show that the closure of the image of $\mathrm{M}(2, e) \rightarrow \mathrm{M}\left(\mathcal{O}_{X}, P_{d}\right)$ is an irreducible component. Because $\mathrm{M}(2, e)$ is itself irreducible, it is enough to show that the image is not contained in $\cup \bar{Z}_{j}$. This follows from a dimension count: $\cup \bar{Z}_{j}$ has dimension equal to $g$, which is less than or equal to $\operatorname{dim} \mathrm{M}(2, e)=4 \bar{g}-3$.

Observe that Theorem 4.7 shows that there are ribbons with the property that every semi-stable rank 2 vector bundle is the specialization of a stable 
generalized line bundle, and there are ribbons that do not have this property. However, it does not fully analyze the case when the inequalities $\bar{g} \geq 2$ and $g>4 \bar{g}-3$ are both satisfied. Thus, it would be interesting to know the answer to the following question:

Question 4.8. Let $X$ be a ribbon such that $\bar{g} \geq 2$ and $g>4 \bar{g}-3$. Does there exist an irreducible component of $\mathrm{M}\left(\mathcal{O}_{X}, P_{d}\right)$ whose general element is a rank 2 vector bundle on $X_{\mathrm{red}}$ ?

We now prove that $\mathrm{M}\left(\mathcal{O}_{X}, P_{d}\right)$ is connected. To establish this, we need another lemma about deformations of generalized line bundles.

Lemma 4.9. Let $X$ be a ribbon and $\mathcal{I}$ a generalized line bundle. If the local index sequence of $\mathcal{I}$ is $\left(b_{1}+1, b_{2}, \ldots, b_{k}\right)$, then $\mathcal{I}$ is the specialization of a generalized line bundle with local index sequence $\left(1, b_{1}, b_{2}, \ldots, b_{k}\right)$.

Proof. The proof is similar to the proof of Lemma 4.5. The essential point is to show that the ideal $\left(\epsilon, s^{b_{1}+1}\right)$ can be deformed to an ideal with local index $b_{1}$ at $\{\epsilon=s=0\}$ and local index 1 at a second point. One such deformation over $k[\alpha]$ is given by the ideal generated by $\epsilon$ and $s^{b_{0}}(s-\alpha)$.

Theorem 4.10. For a ribbon $X$, the moduli space $\mathrm{M}\left(\mathcal{O}_{X}, P_{d}\right)$ is connected.

Proof. The case where $g-2 \bar{g}+1 \leq 0$ can be dispensed with immediately. When this inequality holds, $\mathrm{M}\left(\mathcal{O}_{X}, P_{d}\right)$ is either empty or irreducible by Theorem 4.7. Thus, for the remainder of the proof we assume $g-2 \bar{g}+1>0$.

In this case, we prove connectivity by exhibiting a point lying in every irreducible component. Set $e:=d-g+2 \bar{g}-1$. There are two separate cases to consider: $e$ even and $e$ odd. When $e$ is even, there exist strictly semistable generalized line bundles with local index sequence $(d-e)$. Fix one such sheaf $\mathcal{I}$. We claim that the corresponding point $x_{0}$ of $\mathrm{M}\left(\mathcal{O}_{X}, P_{d}\right)$ lies in every irreducible component of $\mathrm{M}\left(\mathcal{O}_{X}, P_{d}\right)$. Using Theorem 4.6, repeated applications of Lemmas 4.5 and 4.9 show that $x_{0}$ lies in every irreducible component containing a stable generalized line bundle. There is at most one other irreducible component, which, when it exists, parameterizes rank 2 vector bundles on $X_{\text {red }}$. In any case, $x_{0}$ must lie in the locus of rank 2 vector bundles on $X_{\text {red }}$ because $\mathcal{I}$ is Gr-equivalent to such a sheaf (Lemma 3.3). This proves the proposition when $e$ is even.

Now suppose that $e$ is odd. If we replace the strictly semi-stable generalized line bundle with local index sequence $(d-e)$ with a stable generalized line bundle of local index $(d-e-1)$, then a simple modification of the argument given in the $e$ even case shows that there is a point $x_{0}$ lying in every irreducible component that contains a stable generalized line bundle. To complete the proof, we must show that the locus of stable rank 2 vector bundles in $\mathrm{M}\left(X, P_{d}\right)$ has non-empty intersection with the locus of stable generalized line bundles. Because there are no semi-stable rank 2 vector bundles on $\mathbb{P}^{1}$, we may assume $\bar{g} \geq 1$. 
Now suppose first that $g-2 \bar{g}+1=1$. Then there are at most two types of sheaves corresponding to points of $\mathrm{M}\left(\mathcal{O}_{X}, P_{d}\right)$ : stable line bundles of degree $d$ and stable rank 2 vector bundles on $X_{\text {red }}$. By Fact 2.10 , the line bundle locus of fixed degree on $X$ is not proper, hence is not closed in $\operatorname{M}\left(\mathcal{O}_{X}, P_{d}\right)$ because the Simpson moduli space is projective. Thus, the closure of the locus of line bundles must contain at least one point corresponding to a rank 2 vector bundle on $X_{\text {red }}$, which is what we wished to show.

When $g-2 \bar{g}+1>1$, we can reduce to the previous case. Indeed, a suitable blow-up $f: X^{\prime} \rightarrow X$ of $X$ has the property that $g\left(X^{\prime}\right)-2 \bar{g}\left(X^{\prime}\right)+1=1$. We have just shown that there is a family of stable line bundles on $X^{\prime}$ specializing to a stable rank 2 bundle on $X_{\text {red }}^{\prime}=X_{\text {red }}$, and the direct image of this family under $f$ is a family of stable generalized line bundles specializing to a rank 2 vector bundle on $X_{\text {red }}$. In other words, the intersection of the locus of generalized line bundles in $\mathrm{M}\left(\mathcal{O}_{X}, P_{d}\right)$ has non-trivial intersection with the locus of vector bundles on $X_{\text {red }}$, completing the proof.

4.2. Local Structure. We now turn our attention to the local structure of the Simpson moduli space. We compute the tangent space dimension of $\mathrm{M}\left(\mathcal{O}_{X}, P_{d}\right)$ at a point corresponding to a stable sheaf and then apply this result to determine the smooth locus of the moduli space. The specific tangent space computation we give is the following proposition:

Proposition 4.11. Let $x$ be a point of $\mathrm{M}_{\mathrm{s}}\left(\mathcal{O}_{X}, P_{d}\right)$. If $x$ corresponds to a stable generalized line bundle $\mathcal{I}$, then

$$
\operatorname{dim} \mathrm{T}_{x} \mathrm{M}\left(\mathcal{O}_{X}, P_{d}\right)=g+b(\mathcal{I})
$$

If $x$ corresponds to the direct image $i_{*} \mathcal{E}$ of a stable rank 2 vector bundle $\mathcal{E}$ on $X_{\text {red }}$, then

$$
\begin{aligned}
\operatorname{dim} \mathrm{T}_{x} \mathrm{M}\left(\mathcal{O}_{X}, P_{d}\right) & =4 \bar{g}-3+h^{0}\left(X_{\mathrm{red}}, \underline{\operatorname{End}}(\mathcal{E}) \otimes \mathcal{N}^{-1}\right) \\
& =4 g+5-8 \bar{g} \text { if } g \geq 4 \bar{g}-2
\end{aligned}
$$

The proof of the proposition is broken up into two separate lemmas: one computing the dimension when $x$ is a generalized line bundle (Lemma 4.12) and one when $x$ corresponds to a rank 2 vector bundle on $X_{\text {red }}$ (Lemma 4.13).

Let $\mathcal{F}$ be the sheaf parameterized by $x \in \mathrm{M}\left(\mathcal{O}_{X}, P_{d}\right)$. The starting point is the identification of the tangent space of $\mathrm{M}\left(\mathcal{O}_{X}, P_{d}\right)$ at $x$ with the cohomology group $\operatorname{Ext}^{1}(\mathcal{F}, \mathcal{F})$, see e.g., [Har10, Thm. 2.7]. Elements of the tangent space are in natural bijection with morphisms $\operatorname{Spec}\left(k[\alpha] /\left(\alpha^{2}\right)\right) \rightarrow$ $\mathrm{M}\left(\mathcal{O}_{X}, P_{d}\right)$ sending the closed point to $x$. Because $x$ lies in the stable locus, these morphisms in turn are in natural bijection with first-order deformations of $\mathcal{F}$ (i.e. deformations over $\left.k[\alpha] /\left(\alpha^{2}\right)\right)$. The isomophism $\mathrm{T}_{x} \mathrm{M}\left(\mathcal{O}_{X}, P_{d}\right) \cong \operatorname{Ext}^{1}(\mathcal{F}, \mathcal{F})$ is constructed by exhibiting a bijection between first-order deformations of $\mathcal{F}$ and elements of $\operatorname{Ext}^{1}(\mathcal{F}, \mathcal{F})$.

Let $\mathcal{F}_{1}$ be a first order deformation of $\mathcal{F}$, i.e. $\mathcal{F}_{1}$ is a sheaf flat over Spec $k[\alpha] /\left(\alpha^{2}\right)$ whose special fiber is $\mathcal{F}$. Tensoring the short exact sequence 
$k \cong(\alpha) \hookrightarrow k[\alpha] /\left(\alpha^{2}\right) \rightarrow k$ with $\mathcal{F}_{1}$ yields the sequence

$$
\mathcal{F} \cong \alpha \cdot \mathcal{F}_{1} \hookrightarrow \mathcal{F}_{1} \rightarrow \mathcal{F}_{1} / \alpha \cdot \mathcal{F}_{1} \cong \mathcal{F}
$$

This is an extension of $\mathcal{F}$ by $\mathcal{F}$, and hence defines an element $c\left(\mathcal{F}_{1}\right)$ of $\operatorname{Ext}^{1}(\mathcal{F}, \mathcal{F})$. One shows that the rule $\mathcal{F}_{1} \mapsto c\left(\mathcal{F}_{1}\right)$ is well-defined and the induced map

$$
\mathrm{T}_{x} \mathrm{M}\left(\mathcal{O}_{X}, P_{d}\right) \rightarrow \operatorname{Ext}^{1}(\mathcal{F}, \mathcal{F})
$$

is a bijection. As an aside, we remark that when $x$ is strictly semi-stable, there is no longer a canonical identification $\mathrm{T}_{x} \mathrm{M}\left(\mathcal{O}_{X}, P_{d}\right) \cong \operatorname{Ext}^{1}(\mathcal{F}, \mathcal{F})$. Rather, there is a more complicated description of the tangent space involving a natural action of $\operatorname{Aut}(\mathcal{F})$ on $\operatorname{Ext}^{1}(\mathcal{F}, \mathcal{F})$.

We now compute $\operatorname{Ext}^{1}(\mathcal{I}, \mathcal{I})$ for $\mathcal{I}$ a generalized line bundle.

Lemma 4.12. If $\mathcal{I}$ is a generalized line bundle, then we have

$$
\operatorname{dim}\left(\operatorname{Ext}^{1}(\mathcal{I}, \mathcal{I})\right)=g+b(\mathcal{I})+h^{0}\left(X^{\prime}, \mathcal{O}_{X^{\prime}}\right)-1
$$

where $X^{\prime}$ is the associated blow-up of $\mathcal{I}$.

If we additionally assume $2 \bar{g} \leq g$, then this formula simplifies to

$$
\operatorname{dim}\left(\operatorname{Ext}^{1}(\mathcal{I}, \mathcal{I})\right)=g+b(\mathcal{I})
$$

Proof. An inspection of the local-to-global spectral sequence

$$
H^{p}\left(X, \underline{\operatorname{Ext}}^{q}(\mathcal{I}, \mathcal{I})\right) \Rightarrow \operatorname{Ext}^{p+q}(\mathcal{F}, \mathcal{F})
$$

computing Ext shows that there is a short exact sequence

$$
H^{1}(X, \underline{\operatorname{End}}(\mathcal{I})) \hookrightarrow \operatorname{Ext}^{1}(\mathcal{I}, \mathcal{I}) \rightarrow H^{0}\left(X, \underline{\operatorname{Ext}}^{1}(\mathcal{I}, \mathcal{I})\right) .
$$

We prove the proposition by computing the right-most term and the leftmost term in the above sequence. If we write $\mathcal{I}=f_{*}\left(\mathcal{I}^{\prime}\right)$ for a line bundle $\mathcal{I}^{\prime}$ on a blow-up $f: X^{\prime} \rightarrow X$, then the canonical map $f_{*}\left(\mathcal{O}_{X^{\prime}}\right) \rightarrow \underline{\operatorname{End}}(\mathcal{I})$ is an isomorphism (because any $\mathcal{O}_{X^{-}}$linear map $\mathcal{I} \rightarrow \mathcal{I}$ is $f_{*} \mathcal{O}_{X^{\prime}}$-linear). As a consequence, $H^{1}(X, \underline{\operatorname{End}}(\mathcal{I}))=H^{1}\left(X^{\prime}, \mathcal{O}_{X^{\prime}}\right)$, which is of dimension $g-b(\mathcal{I})+h^{0}\left(X^{\prime}, \mathcal{O}_{X^{\prime}}\right)-1$.

We also need to compute $H^{0}\left(X, \underline{\operatorname{Ext}}^{1}(\mathcal{I}, \mathcal{I})\right)$. The sheaf $\underline{\operatorname{Ext}}^{1}(\mathcal{I}, \mathcal{I})$ is supported on the points $p$ satisfying $b_{p}(\mathcal{I})>0$. If we label these points as $p_{1}, \ldots, p_{n}$, then the space of global sections decomposes as

$$
H^{0}\left(X, \underline{\operatorname{Ext}}^{1}(\mathcal{I}, \mathcal{I})\right)=\oplus_{j=1}^{n} \operatorname{Ext}^{1}\left(\mathcal{I}_{p_{j}}, \mathcal{I}_{p_{j}}\right),
$$

The module $\operatorname{Ext}^{1}\left(\mathcal{I}_{p_{j}}, \mathcal{I}_{p_{j}}\right)$ has finite length because $\mathcal{I}$ is locally free away from $p_{1}, \ldots, p_{n}$, so the module is equal to its completion at the maximal ideal of $\mathcal{O}_{X, p_{j}}$, and this module is $\operatorname{Ext}^{1}\left(\widehat{\mathcal{I}}_{p_{j}}, \widehat{\mathcal{I}}_{p_{j}}\right)$.

The completion $\widehat{\mathcal{I}}_{p_{j}}$ is isomorphic to $\mathcal{O}_{b_{p_{j}}}$, and we can compute $\operatorname{Ext}^{1}\left(\widehat{\mathcal{I}}_{p_{j}}, \widehat{\mathcal{I}}_{p_{j}}\right)$ as the first cohomology group of the complex obtained by applying $\operatorname{Hom}\left(\cdot, I_{b_{p_{j}}}\right)$ 
to the free resolution (2.2). A computation shows this cohomology group has dimension $\operatorname{dim} \operatorname{Ext}^{1}\left(\widehat{\mathcal{I}}_{p_{j}}, \widehat{\mathcal{I}}_{p_{j}}\right)=2 b_{p_{j}}(\mathcal{I})$. Summing over all $j$, we have

$$
\begin{aligned}
\operatorname{dim} \operatorname{Ext}^{1}(\mathcal{I}, \mathcal{I}) & =\left(g-b(\mathcal{I})+h^{0}\left(X^{\prime}, \mathcal{O}_{X^{\prime}}\right)-1\right)+\left(2 b_{p_{1}}(\mathcal{I})+\cdots+2 b_{p_{n}}(\mathcal{I})\right) \\
& =g+b(\mathcal{I})+h^{0}\left(X^{\prime}, \mathcal{O}_{X^{\prime}}\right)-1 .
\end{aligned}
$$

This establishes (4.3). When $2 \bar{g} \leq g$, the nilradical has negative degree, and hence no non-zero global sections. In particular, $h^{0}\left(X^{\prime}, \mathcal{O}_{X^{\prime}}\right)-1=0$, proving that (4.4) holds.

We now turn our attention to sheaves of the form $i_{*} \mathcal{E}$.

Lemma 4.13. Let $\mathcal{E}$ be a stable rank 2 vector bundle on $X_{\mathrm{red}}$. Then we have

$$
\operatorname{dim} \operatorname{Ext}^{1}\left(i_{*} \mathcal{E}, i_{*} \mathcal{E}\right)=4 \bar{g}-3+h^{0}\left(X_{\text {red }}, \mathcal{N}^{-1} \otimes \underline{\operatorname{End}}(\mathcal{E})\right) .
$$

If we additionally assume $g \geq 4 \bar{g}-2$, then this formula simplifies to

$$
\operatorname{dim} \operatorname{Ext}^{1}\left(i_{*} \mathcal{E}, i_{*} \mathcal{E}\right)=4 g+5-8 \bar{g} .
$$

Proof. Like the previous lemma, this is proven using a spectral sequence argument. For any two $\mathcal{O}_{X_{\text {red }}}$-modules $\mathcal{A}$ and $\mathcal{B}$, adjunction provides a canonical identification $\operatorname{Hom}_{\mathcal{O}_{X}}\left(i_{*} \mathcal{A}, i_{*} \mathcal{B}\right)=\operatorname{Hom}_{\mathcal{O}_{X_{\text {red }}}}\left(i^{*} i_{*} \mathcal{A}, \mathcal{B}\right)$, hence the groups $\operatorname{Ext}^{n}\left(i_{*} \mathcal{A}, i_{*} \mathcal{B}\right)$ and $\operatorname{Ext}^{n}\left(i^{*} i_{*} \mathcal{A}, \mathcal{B}\right)$ are isomorphic. We compute $\operatorname{Ext}^{1}\left(i_{*} \mathcal{E}, i_{*} \mathcal{E}\right)$ by working with a spectral sequence describing $\operatorname{Ext}^{1}\left(i^{*} i_{*} \mathcal{E}, \mathcal{E}\right)$.

The functor $\operatorname{Hom}\left(i^{*} i_{*}, \mathcal{E}\right)$ is the composition of the functors $G:=i^{*} i_{*}$ and $F:=\operatorname{Hom}_{\mathcal{O}_{X_{\text {red }}}}(, \mathcal{E})$, so there is a Grothendieck spectral sequence:

$$
\operatorname{Ext}^{p}\left(\operatorname{Tor}_{q}\left(i_{*}\left(\mathcal{O}_{X_{\text {red }}}\right), i_{*} \mathcal{E}\right), \mathcal{E}\right) \Rightarrow \operatorname{Ext}^{p+q}\left(i^{*} i_{*} \mathcal{E}, \mathcal{E}\right)
$$

The first four terms of the associated exact sequence of low degree terms are:

$$
\operatorname{Ext}^{1}(\mathcal{E}, \mathcal{E}) \hookrightarrow \operatorname{Ext}^{1}\left(i^{*} i_{*} \mathcal{E}, \mathcal{E}\right) \rightarrow \operatorname{Hom}\left(\operatorname{Tor}_{1}\left(i_{*} \mathcal{O}_{X_{\text {red }}}\right), \mathcal{E}\right) \rightarrow \operatorname{Ext}^{2}(\mathcal{E}, \mathcal{E}) .
$$

Because $X_{\text {red }}$ is a non-singular curve, the last term $\operatorname{Ext}^{2}(\mathcal{E}, \mathcal{E})$ vanishes. We can also compute the second-to-last term. Associated to the short exact sequence $\mathcal{N} \hookrightarrow \mathcal{O}_{X} \rightarrow \mathcal{O}_{X_{\text {red }}}$ is a long exact sequence of $\operatorname{Tor}\left(\ldots, i_{*} \mathcal{E}\right)$-groups whose connecting map

$$
\partial: \operatorname{Hom}(\mathcal{N} \otimes \mathcal{E}, \mathcal{E}) \rightarrow \operatorname{Hom}\left(\operatorname{Tor}_{1}\left(i_{*} \mathcal{O}_{X_{\text {red }}}, i_{*} \mathcal{E}\right)\right)
$$

is an isomorphism. We now compute the dimension of $\operatorname{Ext}^{1}\left(i_{*} \mathcal{E}, i_{*} \mathcal{E}\right)$ using Sequence (4.7):

$$
\begin{aligned}
\operatorname{dim} \operatorname{Ext}^{1}\left(i_{*} \mathcal{E}, i_{*} \mathcal{E}\right) & =\operatorname{dim} \operatorname{Ext}^{1}\left(i^{*} i_{*} \mathcal{E}, \mathcal{E}\right) \\
& =\operatorname{dim} \operatorname{Ext}^{1}(\mathcal{E}, \mathcal{E})+\operatorname{dim} \operatorname{Hom}(\mathcal{N} \otimes \mathcal{E}, \mathcal{E}) \\
& =h^{1}\left(X_{\text {red }}, \underline{\operatorname{End}}(\mathcal{E})\right)+h^{0}\left(X_{\text {red }}, \mathcal{N}^{-1} \otimes \underline{\operatorname{End}}(\mathcal{E})\right) .
\end{aligned}
$$


Note that $\operatorname{Hom}(\mathcal{E}, \mathcal{E})=H^{0}\left(X_{\text {red }}, \underline{\operatorname{End}}(\mathcal{E})\right)$ is 1-dimensional as $\mathcal{E}$ is stable. Moreover, $\underline{\operatorname{End}}(\mathcal{E})$ is of rank 4 and degree 0 . Hence by the definition of degree of vector bundles on curves ([LP95, Sec. 2.6]) we have

$$
\chi\left(X_{\text {red }}, \underline{\operatorname{End}}(\mathcal{E})\right)=4 \cdot(1-\bar{g})+0=4-4 \bar{g} .
$$

It follows that

$$
h^{1}\left(X_{\text {red }}, \underline{\operatorname{End}}(\mathcal{E})\right)=1-(4-4 \bar{g})=4 \bar{g}-3 .
$$

Altogether this proves Formula (4.5).

Now assume $g \geq 4 \bar{g}-2$. Because $\mathcal{E}$ is stable, the rank 4 vector bundle $\mathcal{N}^{-1} \otimes \underline{\operatorname{End}}(\mathcal{E})$ is semi-stable ([Laz04, Cor. 6.4.14]) of degree $4+4 g-8 \bar{g}$. Therefore, we have

$$
\chi\left(X_{\text {red }}, \mathcal{N}^{-1} \otimes \underline{\operatorname{End}}(\mathcal{E})\right)=4 \cdot(1-\bar{g})+(4+4 g-8 \bar{g})=8+4 g-12 \bar{g} .
$$

Using Serre duality on $X_{\text {red }}$, one checks that $h^{1}\left(X_{\text {red }}, \mathcal{N}^{-1} \otimes \underline{\operatorname{End}}(\mathcal{E})\right)=0$. We thus conclude that

$$
\operatorname{dim} \operatorname{Ext}^{1}\left(i_{*} \mathcal{E}, i_{*} \mathcal{E}\right)=(4 \bar{g}-3)+(8+4 g-12 \bar{g})=4 g+5-8 \bar{g},
$$

completing the proof of Formula (4.6).

One immediate corollary of the lemma is the following:

Corollary 4.14. Let $X$ be a ribbon. If $\bar{g} \geq 2$ and $g \geq 4 \bar{g}-2$, then the smooth locus of $\mathrm{M}\left(\mathcal{O}_{X}, P_{d}\right)$ is equal to the open subset parameterizing line bundles on $X$.

Proof. First, let us prove the weaker claim concerning the smooth locus of the space $\mathrm{M}_{\mathrm{s}}\left(\mathcal{O}_{X}, P_{d}\right)$ of stable sheaves. It is enough to show that if $x \in \mathrm{M}_{\mathrm{S}}\left(\mathcal{O}_{X}, P_{d}\right)$, then the tangent space dimension of $\mathrm{M}\left(\mathcal{O}_{X}, P_{d}\right)$ equals the local (topological) dimension if and only if $x$ corresponds to a line bundle. Theorems 4.6, 4.7 together with Proposition 4.11 show that equality must fail except possibly in the following cases: when $x$ corresponds to a stable line bundle and when $x$ corresponds to a stable rank 2 vector bundle on $X_{\text {red. }}$. We must show that the second case cannot occur.

If $x$ corresponds to a stable rank 2 vector bundle on $X_{\text {red }}$, then the tangent space dimension is:

$$
\operatorname{dim} \mathrm{T}_{x} \mathrm{M}_{\mathrm{s}}\left(\mathcal{O}_{X}, P_{d}\right)=4 g+5-8 \bar{g} .
$$

We have not computed the local dimension of $\mathrm{M}\left(\mathcal{O}_{X}, P_{d}\right)$ at $x$, but Theorems 4.6 and 4.7 show this local dimension at $x$ is either $g$ or $4 \bar{g}-3$. Using the assumption $\bar{g} \geq 2$ and $g \geq 4 \bar{g}-2$, a direct computation shows that the local dimension at $x$ is strictly smaller than $4 g+5-8 \bar{g}$, proving the claim concerning the locus of stable sheaves.

What about the strictly semi-stable locus? Because $\bar{g} \geq 2$, every strictly semi-stable point is the specialization of a stable point that is not a line bundle. Indeed, the strictly semi-stable locus is contained in the image of $\mathrm{M}(2, e)$, and the stable locus is dense in $\mathrm{M}(2, e)$. Because the singular locus 
is closed, we can conclude that the strictly semi-stable locus is contained in the singular locus, completing the proof.

Observe that the hypothesis that $g \geq 4 \bar{g}-2$ is used to compute the dimension of the tangent space of $\mathrm{M}\left(\mathcal{O}_{X}, P_{d}\right)$ at a point corresponding to a stable rank 2 vector bundle on $X_{\text {red }}$. The method of proof can be used to describe the singular locus of $\mathrm{M}\left(\mathcal{O}_{X}, P_{d}\right)$ under weaker hypotheses, but then the conclusion becomes more difficult to state: If $g \leq 4 \bar{g}-3$ is allowed, then the smooth locus may contain stable rank 2 vector bundles. For example, if $g$ is sufficiently negative and $\bar{g} \geq 2$, then there are no semi-stable generalized line bundles, so $\mathrm{M}\left(\mathcal{O}_{X}, P_{d}\right)$ has a unique component of dimension $4 \bar{g}-3$ whose smooth locus contains the locus of stable rank 2 vector bundles on $X_{\text {red }}$. The proof also makes use of the fact that every strictly semi-stable sheaf is the specialization of a stable sheaf. In general, this condition may fail to hold (e.g. $\bar{g}=1$ and $g=2$ ). When this occurs, a more delicate analysis of the semi-stable locus is needed.

\section{REFERENCES}

[AK79] Allen B. Altman and Steven L. Kleiman, Compactifying the Picard scheme. II, Amer. J. Math. 101 (1979), no. 1, 10-41. MR 527824 (81f:14025b)

[BE95] Dave Bayer and David Eisenbud, Ribbons and their canonical embeddings, Trans. Amer. Math. Soc. 347 (1995), no. 3, 719-756. MR 1273472 (95g:14032)

[BLR90] Siegfried Bosch, Werner Lütkebohmert, and Michel Raynaud, Néron models, Ergebnisse der Mathematik und ihrer Grenzgebiete (3) [Results in Mathematics and Related Areas (3)], vol. 21, Springer-Verlag, Berlin, 1990. MR 1045822 (91i:14034)

[Che10] Dawei Chen, Linear series on ribbons, Proc. Amer. Math. Soc. 138 (2010), no. 11, 3797-3805. MR 2679602 (2011j:14071)

[DEL97] Ron Donagi, Lawrence Ein, and Robert Lazarsfeld, Nilpotent cones and sheaves on K3 surfaces, Birational algebraic geometry (Baltimore, MD, 1996), Contemp. Math., vol. 207, Amer. Math. Soc., Providence, RI, 1997, pp. 51-61. MR 1462924 (98f:14006)

[Dré06] Jean-Marc Drézet, Faisceaux cohérents sur les courbes multiples, Collect. Math. 57 (2006), no. 2, 121-171. MR 2223850 (2007b:14077)

[Dré08] Moduli spaces of coherent sheaves on multiples curves, Algebraic cycles, sheaves, shtukas, and moduli, Trends Math., Birkhäuser, Basel, 2008, pp. 33-43. MR 2402692 (2009e:14015)

[Dré09] - Faisceaux sans torsion et faisceaux quasi localement libres sur les courbes multiples primitives, Math. Nachr. 282 (2009), no. 7, 919-952. MR 2541242 (2010j:14027)

[Dré11] Sur les conditions d'existence des faisceaux semi-stables sur les courbes multiples primitives, Pacific J. Math. 249 (2011), no. 2, 291-319.

[EG95] David Eisenbud and Mark Green, Clifford indices of ribbons, Trans. Amer. Math. Soc. 347 (1995), no. 3, 757-765. MR 1273474 (95g:14033)

[Har10] Robin Hartshorne, Deformation theory, Graduate Texts in Mathematics, 257, Springer, New York, 2010. MR 2583634 (2011c:14023)

[Ina04] Michi-Aki Inaba, On the moduli of stable sheaves on some nonreduced projective schemes, J. Algebraic Geom. 13 (2004), no. 1, 1-27. MR 2008714 (2004h:14020)

[Kas13] Jesse Leo Kass, Two ways to degenerate the Jacobian are the same, Algebra Number Theory 7 (2013), no. 2, 379-404. MR 3123643 
[Lan04] Adrian Langer, Semistable sheaves in positive characteristic, Ann. Math. (2) 159 (2004), no. 1, 251-276. MR 2051393 (2005c:14021)

[Laz04] Robert Lazarsfeld, Positivity in algebraic geometry. II, Ergebnisse der Mathematik und ihrer Grenzgebiete. 3. Folge. A Series of Modern Surveys in Mathematics [Results in Mathematics and Related Areas. 3rd Series. A Series of Modern Surveys in Mathematics], vol. 49, Springer-Verlag, Berlin, 2004, Positivity for vector bundles, and multiplier ideals. MR 2095472 (2005k:14001b)

[LLR04] Qing Liu, Dino Lorenzini, and Michel Raynaud, Néron models, Lie algebras, and reduction of curves of genus one, Invent. Math. 157 (2004), no. 3, 455-518. MR 2092767 (2005m:14039)

[LP95] Joseph Le Potier, Fibrés vectoriels sur les courbes algébriques, Publications Mathématiques de l'Université Paris 7-Denis Diderot [Mathematical Publications of the University of Paris 7-Denis Diderot], 35, Université Paris 7-Denis Diderot U.F.R de Mathématiques, Paris, 1995, With a chapter by Christoph Sorger. MR 1370930 (97c:14034)

[Mar96] Masaki Maruyama, Construction of moduli spaces of stable sheaves via Simpson's idea, Moduli of vector bundles (Sanda, 1994; Kyoto, 1994), Lecture Notes in Pure and Appl. Math., vol. 179, Dekker, New York, 1996, pp. 147-187. MR 1397986 (97h:14020)

[Ray70] M. Raynaud, Spécialisation du foncteur de Picard, Inst. Hautes Études Sci. Publ. Math. (1970), no. 38, 27-76. MR 0282993 (44 \#227)

[Sim94] Carlos T. Simpson, Moduli of representations of the fundamental group of a smooth projective variety. I, Inst. Hautes Études Sci. Publ. Math. (1994), no. 79, 47-129. MR 1307297 (96e:14012)

[Yan03] Jin-Gen Yang, Coherent sheaves on a fat curve, Japan. J. Math. (N.S.) 29 (2003), no. 2, 315-333. MR 2035543 (2005c:14022)

Department of Mathematics, Boston College, Chestnut Hill, MA 02467, USA

E-mail address: dawei.chen@bc.edu

Department of Mathematics, University of South Carolina, Columbia, South CARolina 29208, USA

E-mail address: kassj@math.sc.edu 